\title{
The difference engine: A model of diversity in speeded cognition
}

\author{
JOEL MYERSON and SANDRA HALE \\ Washington University, St. Louis, Missouri \\ YINGYE ZHENG \\ University of Washington, Seattle, Washington \\ LISA JENKINS \\ Wyeth Research, Collegeville, Pennsylvania \\ and \\ KEITH F. WIDAMAN \\ University of California, Davis, California
}

\begin{abstract}
A theory of diversity in speeded cognition, the difference engine, is proposed, in which information processing is represented as a series of generic computational steps. Some individuals tend to perform all of these computations relatively quickly and other individuals tend to perform them all relatively slowly, reflecting the existence of a general cognitive speed factor, but the time required for response selection and execution is assumed to be independent of cognitive speed. The difference engine correctly predicts the positively accelerated form of the relation between diversity of performance, as measured by the standard deviation for the group, and task difficulty, as indexed by the mean response time (RT) for the group. In addition, the difference engine correctly predicts approximately linear relations between the RTs of any individual and average performance for the group, with the regression lines for fast individuals having slopes less than 1.0 (and positive intercepts) and the regression lines for slow individuals having slopes greater than 1.0 (and negative intercepts). Similar predictions are made for comparisons of slow, average, and fast subgroups, regardless of whether those subgroups are formed on the basis of differencesin ability, age, or health status. These predictions are consistent with evidence from studies of healthy young and older adults as well as from studies of depressed and age-matched control groups.
\end{abstract}

The results of a number of studies suggest that much of the variance in individual speeded performance, at least on laboratory tasks, may be explained by a single general factor on which diverse tasks load approximately equally (Hale \& Jansen, 1994; Hale \& Myerson, 1993; Zheng, Myerson, \& Hale, 2000; for further examples, see Table 1 in Faust, Balota, Spieler, \& Ferraro, 1999). This finding implies that if a task is speeded, its other characteristics may be much less important, at least for the purpose of predicting the position of an individual's score in the overall distribution of performances (i.e., for predicting performance in standard deviation $[S D]$ units). Even if most of

J.M. and S.H. were partially supported by Grants AG10197 and AG12996 to S.H. from the National Institute on Aging. L.J. was supported by National Institute on Aging training grant AG00030 to Martha Storandt. We thank David Adams, Lisa Emery, and Shannon Robertson for helpful comments on an earlier version. For inspiration, J.M. would like to acknowledge Charles Babbage, John Crowley, William Gibson, and Bruce Sterling. Correspondence should be addressed to J. Myerson, Department of Psychology, Campus Box 1125, Washington University, St. Louis, MO 63130 (e-mail: jmyerson@ artsci.wustl.edu). the variance in individual performance in $S D$ units can be explained by a single factor, however, important questions regarding individual differences still remain. For example, what task characteristics determine how many seconds or milliseconds will separate the performance of the fastest and the slowest individuals, and what mechanism or mechanisms underlie the observed variations in the absolute size of individual differences from task to task?

A recent study by Hale and Jansen (1994) provides an important clue regarding the answers to these questions. They tested 40 young adults on seven different visuospatial information-processing tasks, ranging in difficulty from choice reaction time (choice RT) to mental paper folding. All seven tasks loaded heavily on a general speed factor (first principal component) that accounted for nearly two thirds of the total standardized variance. In order to examine how the differences between the performance of fast, average, and slow individuals changed across tasks, individuals were ranked on the basis of their mean $z$ scores, and the group was divided into quartiles on the basis of these rankings.

When the fastest quartile's mean response times (RTs) for each of the 21 experimental conditions were regressed 


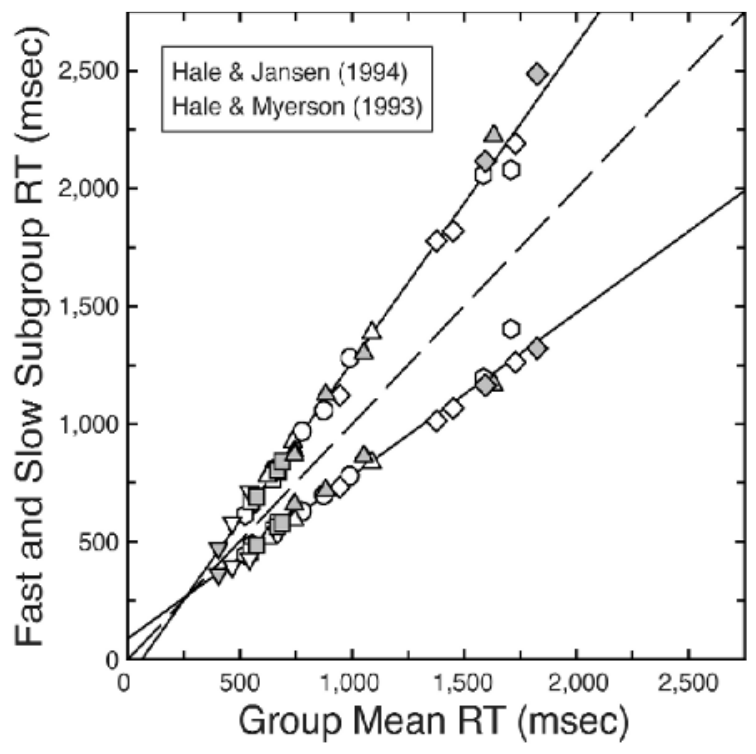

Figure 1. Response times (RTs) of fast and slow subgroups plotted as a function of the group mean RTs. White and gray symbols represent the data from Hale and Jansen (1994) and Hale and Myerson (1993), respectively. Each point represents performance by one subgroup in one experimental condition of one study. In order of increasing task difficulty, the tasks were as follows: inverted triangles, choice RT; octagons, line-length discrimination; squares, letter classification; circles, mental rotation; upright triangles, visual search; octagons, mental paper folding; diamonds, abstract matching. Each regression line represents the fit to the RTs of the corresponding subgroups (i.e., both fast or both slow subgroups) in both studies. If a subgroup mean RT for a particular task condition did not differ from the corresponding group mean RT, the data point for that condition would fall along the dashed diagonal line.

on the corresponding mean RTs for the group as a whole, the regression line accounted for more than $98 \%$ of the variance. Regressing the slowest quartile's mean RTs on the group mean RTs revealed an equally precise relationship (Hale \& Jansen, 1994). Similarly precise relationships were observed when the mean RTs for the fastest and slowest quartiles were regressed on average RTs as defined by the mean for the two center quartiles; again, both $r^{2} \mathrm{~s}$ were greater than .98. As may be seen in Figure 1, the consequence of these linear relations is that the difference between the RTs of the subgroups of fast and slow processors increases with the average RT.

Because of the strong theoretical implications of these findings, we followed the old adage, "measure twice-cut once," and assessed their reliability using another, larger, data set. To this end, we combined the data from young adult controls in previous unpublished and published studies of cognitive aging and development. The RTs of 65 young adults, each of whom had been tested on four tasks (i.e., choice RT, letter classification, visual search, and abstract matching), were reanalyzed using the Hale and Jansen (1994) approach. Again, we found that a principal components analysis of RTs revealed a single general speed factor on which all four tasks loaded heavily and that accounted for a large proportion $(75 \%)$ of the total standardized variance (Hale \& Myerson, 1993).

Following Hale and Jansen (1994), we selected fast and slow subgroups (bottom and top quartiles) from these 65 subjects on the basis of their mean $z$ scores. Notably, 15 of the 16 members of the fast group were faster than average on all four tasks (the other member was faster than average on three of the tasks), and 15 of the 16 slow group members were slower than average on all four tasks (the other member was slower than average on three tasks). When the mean RTs from the two subgroups were regressed on average RTs, extremely orderly linear relations were observed. As was the case with the Hale and Jansen data, this was true regardless of whether average was defined as the mean for the group as a whole or as the mean for the center quartiles. In fact, as may be seen in Figure 1, the data points representing the Hale and Myerson (1993) data set are collinear with the points representing the Hale and Jansen data set.

Further confirmation of this pattern comes from reanalysis of a study by Vernon and Jensen (1984) that used very different procedures and samples. These authors studied two large samples recruited from students attending either a vocational college or a university. All subjects were tested on eight different speeded information-processing tasks, including choice RT, short-term memory scanning,

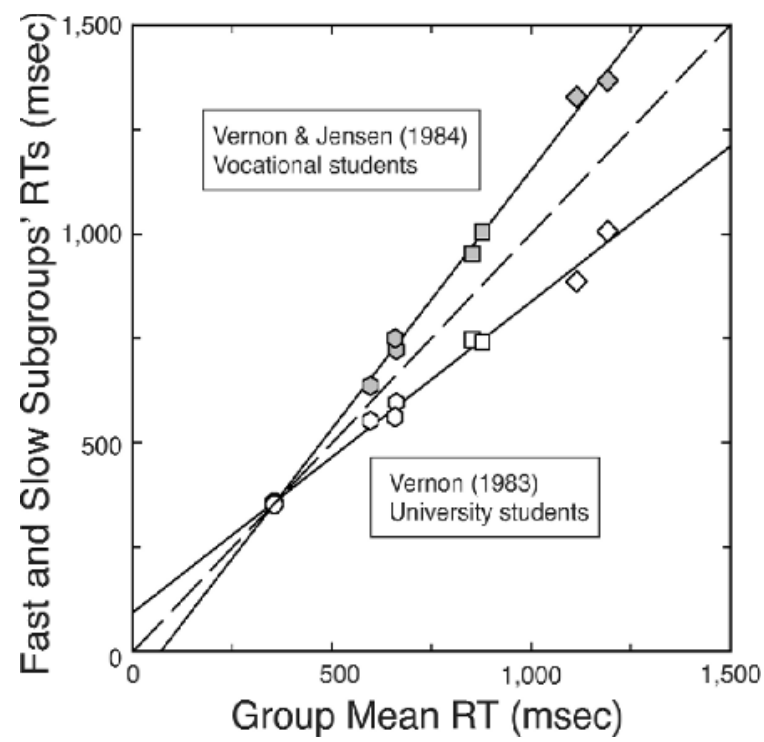

Figure 2. Response times (RTs) of university and vocational college subgroups plotted as a function of the combined student group mean RTs. White and gray symbols represent the data from Vernon (1983) and Vernon and Jensen (1984), respectively. Each point represents performance by one subgroup in one experimental condition of one study. In order of increasing task difficulty, the tasks were as follows: circles, choice RT (Jensen apparatus); octagons, Sternberg memory scanning; squares, same/ different word judgment; diamonds, synonym/antonym judgment. If a subgroup mean $\mathrm{RT}$ for a particular task condition did not differ from the corresponding group mean RT, the data point for that condition would fall along the dashed diagonal line. 
and same/different word and synonym/antonym judgments. For purposes of clarifying the analogy to the two studies considered previously (Hale \& Jansen, 1994; Hale \& Myerson, 1993), the two samples may be thought of as subgroups of a larger group of young adult students. As may be seen in Figure 2, when the RTs for each subgroup are plotted as a function of the mean RT for the whole group, the Vernon and Jensen results are strikingly similar to those of Hale and her colleagues (Figure 1).

Vernon and Jensen (1984) attributed the differences in information-processing speed between the vocational and university students to the fact that the two samples differ in academic ability or psychometric $g$. In support of this claim they noted that as task difficulty increases, the difference between the RTs of the two samples increases. Figure 2 clearly illustrates this finding, assuming that the mean RT for the whole group provides an index of task difficulty. It should be noted, however, that Figure 1 shows the same pattern of RT differences between fast and slow subgroups of students, all of whom attended the same highly competitive university. Regardless of the strength or validity of the association between speed and intelligence, the Vernon and Jensen data taken together with the data from Hale and her colleagues (Hale \& Jansen, 1994; Hale \& Myerson, 1993) suggest that the relationship we have observed between the RTs of fast and slow subgroups is not peculiar to one particular battery of RT tasks, nor does it depend on a specific method for dividing a group into slow and fast subgroups (e.g., top and bottom quartiles or type of college attended) or a particular method for estimating average performance (e.g., overall mean or mean for center two quartiles).

We hypothesize that the observed orderly increase in the difference between slow and fast RTs typifies the effect of task difficulty (indexed by the average RT for the group) on the absolute size of individual differences in performance of speeded tasks. In subsequent sections, we will propose a model to explain this phenomenon and assess its ability to account for both the differences between fast and slow groups as well as differences in individual performance. First, however, it is important to show that even at the subgroup level, the Hale and Jansen (1994) and Hale and Myerson (1993) results are not what would be expected on the basis of the method of constructing subgroups alone, nor do they result simply from a positive relationship between group mean RT and $S D$. To demonstrate this and to examine the role of correlations between RTs on different tasks, we conducted three series of computer simulations.

With respect to the method by which we have constructed subgroups, it is obvious, of course, that selecting fast and slow individuals on the basis of their mean $z$ scores would tend to produce mean RTs for each subgroup that would be smaller and larger, respectively, than average RTs. However, this method would not necessarily lead to a systematic change in the difference between fast and slow RTs as a function of task difficulty of the kind observed in actual data (see Figures 1 and 2). To demonstrate that this is true, we conducted 10 simulations in which RTs were randomly selected for 65 simulated subjects in 10 different task conditions so as to produce the same condition means as those in the Hale and Myerson (1993) study. For all task conditions, the $S D$ was set at $148 \mathrm{msec}$ (the median of the actual $S D$ s for the 10 conditions), and RTs in different task conditions were uncorrelated. Simulated subjects were then sorted into quartiles on the basis of their mean $z$ scores, and the means for the top and bottom quartiles were regressed on overall group means. Across the 10 simulations in this series, the mean of the slopes for the simulated fast quartiles was 1.00, and the mean of the slopes for the simulated slow quartiles was also 1.00 , with standard errors of 0.01 in both cases. Results for a typical simulation (the one with fast and slow slopes closest in value to the means) are shown in the upper left panel of Figure 3. As may be seen, the size of the difference between the RTs of the fast and slow subgroups was very small and remained approximately constant across tasks.

Notably, the method of selecting fast and slow quartiles did not produce subgroups in which individual simulated subjects were consistently fast or slow. In repeated simulations, most simulated subjects from the top and bottom quartiles were faster or slower than the mean in only 6 or 7 task conditions (mean for the representative simulation depicted was 6.50), just slightly more than the 5 out of 10 expected for those of average speed. In contrast, the actual fast and slow subjects in the Hale and Myerson (1993) study were very consistent. Specifically, all but 1 subject from the bottom or top quartile was faster or slower than average in at least 9 out of 10 conditions of the four tasks.

Further, the systematic increase in the difference between the RTs of fast and slow subgroups with task difficulty observed in the Hale and Jansen (1994) and Hale and Myerson (1993) data is not simply a consequence of a positive relationship between mean and $S D$ (Hale, Myerson, Smith, \& Poon, 1988). To demonstrate this, we performed a second set of simulations similar to those just described except that both the mean RTs and the $S D$ s for the 10 task conditions were set equal to those in the corresponding 10 conditions from the Hale and Myerson (1993) study. Because of the positive relationship between mean and $S D$, this set of simulations will be referred to as the dependent $S D$ simulations to distinguish them from the previous set of independent $S D$ simulations. As in the independent $S D$ simulations, the correlation between RTs on different tasks was set to zero, and the 65 simulated subjects were again sorted into quartiles on the basis of their mean $z$ scores.

With the observed relationship between mean RT and $S D$ incorporated into the simulation, the difference between the mean RTs for the fast and slow quartiles did increase slightly with task difficulty. The rate of increase, however, was substantially less than that observed in the real data. To compare the actual and simulated results, we conducted 10 complete simulations in order to estimate the slopes for the regression of RTs of the fast and slow quartiles on the simulated group mean RTs. The mean of the slopes for the simulated slow quartiles was 1.14 (with a 


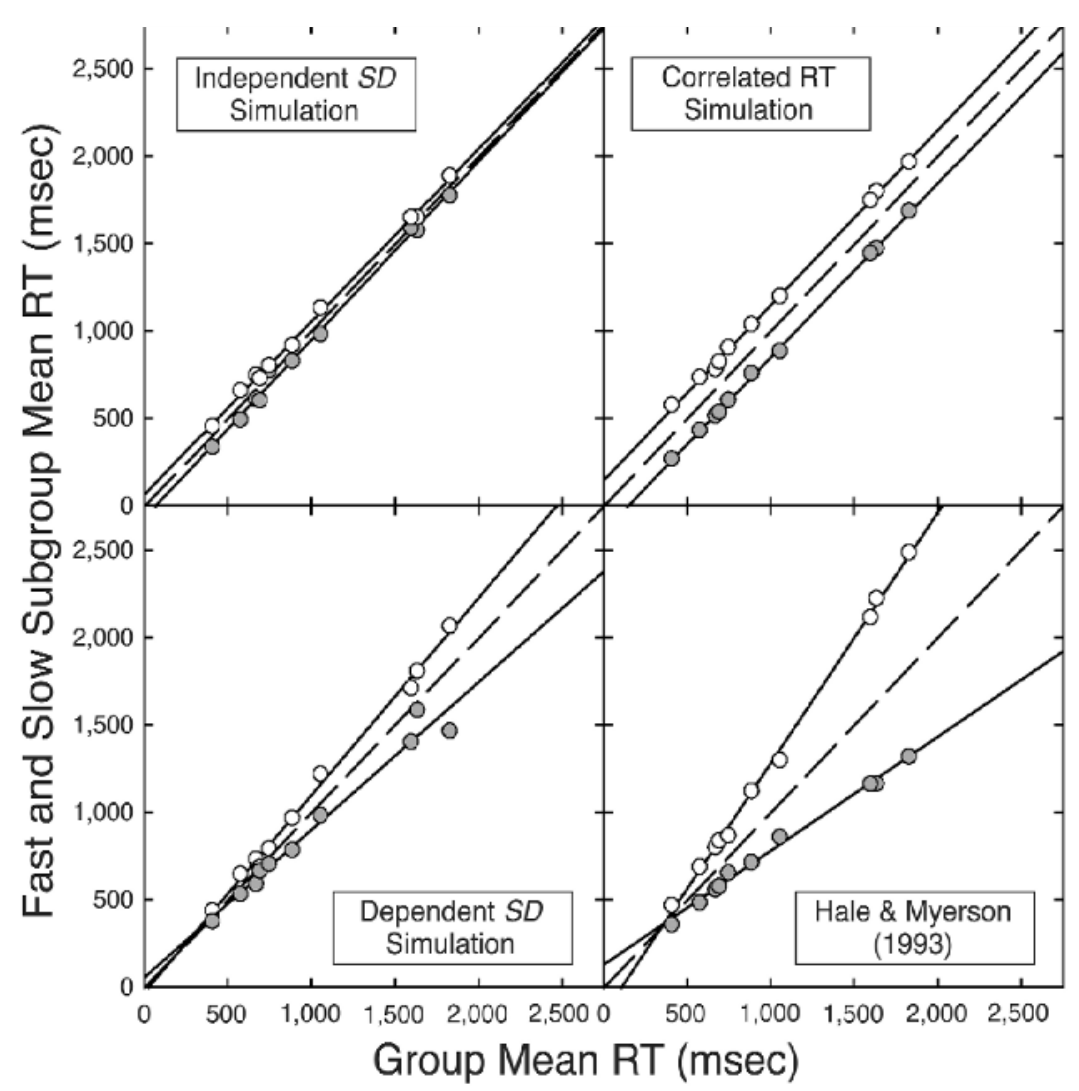

Figure 3. Simulated and actual fast and slow subgroups' mean response times (RTs) plotted as a function of group mean RTs. The two upper panels and the lower left panel present data generated by three different computer simulations (see text for description). The lower right panel presents the data from Hale and Myerson (1993) for comparison purposes. In all panels, the white and gray circles represent the slow and fast subgroups, respectively.

standard error of 0.02), which is considerably less than the slope of 1.45 observed for the slow subgroup in the Hale and Myerson (1993) experiment. The mean of the slopes for the simulated fast quartiles was 0.85 (standard error of 0.02 ), which is greater than the slope of 0.65 for the actual fast subgroup. Thus, for every increase of $100 \mathrm{msec}$ in task difficulty (as measured by group mean RT), the difference between the RTs of the actual fast and slow subgroups increased by $80 \mathrm{msec}$. This is more than 2.5 times the rate of increase in the difference between the simulated subgroups (29 $\mathrm{msec}$ per $100 \mathrm{msec}$ increase in difficulty). The results for a representative dependent $S D$ simulation (the one with fast and slow slopes closest in value to the means for the 10 simulations) are shown in the lower left panel of Figure 3 and may be compared with the actual Hale and Myerson results shown in the lower right panel.

As in our set of independent $S D$ simulations, there was relatively little consistency in performance at the individual level in the set of dependent $S D$ simulations despite the method of selecting fast and slow quartiles. Most simulated fast and slow subjects were faster than average or slower than average, respectively, in only 6 or 7 task con- ditions, just slightly more than the 5 out of 10 expected for those of average speed. In the typical dependent $S D$ simulation depicted, the mean number of conditions with RTs appropriately faster or slower than average was 6.75 , which may be compared with a mean of 9.66 out of 10 for the actual fast and slow subjects in the Hale and Myerson (1993) study.

We also conducted a third set of 10 simulations in which RTs in different conditions were correlated in order to assess how individual consistency in performance affects the pattern of differences between the RTs of fast and slow subgroups. For these correlated $R T$ simulations, correlations between RTs for different task conditions were produced by adding condition-specific random variables to an individual-specific random variable (for which individual values were held constant across task conditions). Condition mean RTs were set equal to those observed in the Hale and Myerson (1993) study, and SDs were set equal to the median of the observed $S D \mathrm{~s}$. As was the case for the actual subjects, most simulated fast or slow subjects were consistently fast or slow (i.e., 9 or 10 conditions faster or slower than average; for the most representative simula- 
tion, $M=9.39$ out of 10). Unlike the results of the actual study, however, the mean regression slopes for both the simulated fast and the simulated slow subgroups were 1.00 (standard errors less than .01). The fast and slow subgroup regression results for the most representative correlated RT simulation (i.e., the one whose regression slopes were closest to the mean slopes) are shown in the upper right panel of Figure 3.

Comparison of the Hale and Myerson (1993) results (lower right panel of Figure 3) with the results from our three sets of simulations (i.e., the independent $S D$, dependent $S D$, and correlated RT simulations) clearly reveals that none of the alternatives in question - the method of creating fast and slow subgroups based on mean $z$ scores, the empirical relationship between group means and $S D$ s, or the observed correlations between RTs-are sufficient in and of themselves to explain the observed pattern of differences between actual fast and slow subjects. The simulation results do suggest, however, possible roles for both correlations between RTs in different task conditions and a positive relationship between condition mean RT and $S D$. It is possible, moreover, that consistently fast or slow individual performance may enhance patterns of differences between fast and slow subgroups (compare the independent $S D$ and correlated RT simulations), and that the relationship between group mean and $S D$ may provide the basic pattern to be enhanced (compare the independent $S D$ and dependent $S D$ simulations). However, the existence of intertask correlations, on the one hand, and a positive relationship between mean RT and $S D$, on the other, may not necessarily reflect separate empirical constraints. Rather, as we will propose in subsequent sections, a general cognitive speed factor may play a fundamental role in determining the relationship between mean and $S D$ as well as the difference between fast and slow subgroups or individuals. This view is fundamental to our theoretical account of the mechanism that determines the absolute size of individual differences on different tasks.

Before proceeding to the theoretical development, however, we wish to point out that the present concern extends beyond differences in cognitive speed between healthy individuals of the same age. The present effort is also motivated by the fact that a growing number of studies involving a variety of special populations have reported findings related to those just discussed, suggesting that the mechanism underlying variation in the absolute size of individual differences in RTs on various cognitive tasks may have a great deal in common with the mechanism that governs the size of group differences in RTs on such tasks.

More specifically, when group differences are assessed across multiple tasks, the relations between the RTs of one group and those of another (e.g., control) group are often well described by linear functions similar to those we have observed between the RTs of same-age individuals who differ in ability. For example, such relations have been reported when children and older adults are compared with young adults (for a review, see Cerella \& Hale, 1994). Recently, similar results have been observed in studies com- paring the performance of groups with a variety of conditions, including mental retardation (Kail, 1992), clinical depression (White, Myerson, \& Hale, 1997), closed-head injury (Ferraro, 1996), multiple sclerosis (Kail, 1997), and Alzheimer's disease (Myerson, Lawrence, Hale, Jenkins, \& Chen, 1998), with the performance of appropriate controls. In all of the cited studies, the difference between the RTs of affected groups and appropriate controls increased systematically with task difficulty as indexed by the RT of the control group.

The orderly functional relations seen in these studies of the speeded cognitive performance of various groups appear to be strikingly similar to those seen in our studies of the diversity of performance within groups (Hale \& Jansen,
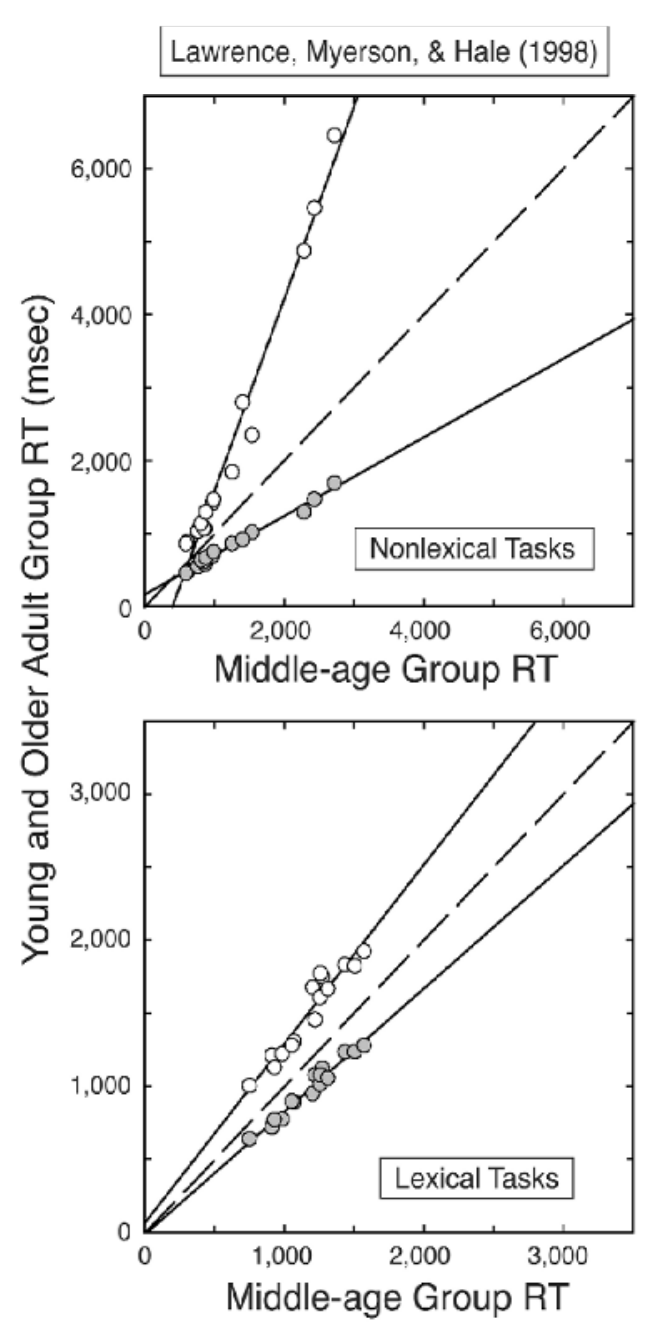

Figure 4. Nonlexical and lexical response times (RTs) of young and older adult groups plotted as a function of the corresponding RTs of a middle-age group. Data are taken from Lawrence, Myerson, and Hale (1998). In both the upper and lower panels, white circles represent data from the older adult group (80- to 89year-olds), and gray circles represent data from the young adult group (18- to 21-year-olds). The middle-age group consisted of 50- to 59-year-olds. 
1994; Hale \& Myerson, 1993; Zheng et al., 2000). In both cases, the RTs of one set of individuals tested on a number of quite different tasks can be predicted directly from the RTs of another set of individuals without taking the nature of the tasks into account (other than whether the information that was processed was verbal or spatial). The parallel may be seen most clearly in Figure 4, in which the group mean RTs of young adults and adults in their 80s are each plotted as a function of a group of intermediate age, adults in their 50s (data are taken from Lawrence, Myerson, \& Hale, 1998). In both the verbal and spatial domains, group differences increased systematically with increases in task difficulty.

Such findings suggest that, as with individual differences and differences between fast and slow subgroups, there may be a general speed factor that underlies the differences observed between the RTs of various groups, at least within each processing domain (i.e., verbal or spatial) (e.g., Cerella, 1985, 1990; Hale, Myerson, \& Wagstaff, 1987; Kail, 1991). Although orderly relations between the mean RTs of different groups can be misleading under certain circumstances (Fisher \& Glaser, 1996), such relations nevertheless raise the possibility that basic models developed to explain individual differences in ability may also apply to other individual difference variables (e.g., age and health status), and perhaps to the interaction of such variables as well.

In this paper, we develop a model of the interaction between individual differences in the ability to process information rapidly, on the one hand, and the amount of processing required by different tasks, on the other hand. We then apply this model to the problem of how individual ability and task difficulty interact with other individual difference variables such as age and health status. There is a rich database with respect to speeded cognition in young and older adults that exemplifies the issues involved in such interactions, and such data provide a test case with which to evaluate our model. We would emphasize, however, that our ultimate claim is more general and applies not just to individualdifferences and aging, but also to developmental changes in processing speed as well as to differences between healthy individuals and those affected by various health conditions.

In developing our model, we will employ simplifying assumptions that ignore distinctions that may be the focus of other theoretical and experimental efforts. We will do this for two reasons: first, because of differences in purpose between the present effort and other research, and second, because of differences in scale. These two reasons are obviously not mutually exclusive. After all, different purposes may require measures and analyses that provide different degrees of resolution. For example, trying to draw (or use) a full-scale map of the world (i.e., one in which 1 foot represents 1 foot) obviously would create problems. There is usually a tradeoff between resolution and scope, and people tend to select the type of map with the degree of resolution appropriate for the purpose at hand, using both more detailed maps of smaller areas (e.g., cities) and less detailed maps of larger areas (e.g., countries).
The focus of the present effort is on predicting performance over a range of task difficulty that spans an order of magnitude (i.e., 500-5,000 msec), at least for some groups (e.g., older adults), and differences of an order of magnitude or smaller (i.e., on the order of $50 \mathrm{msec}$ or less) may be neglected in the process. Just as makers and users of maps of whole countries would not claim that maps of individual cities are wrong, so our use of relatively low degrees of resolution in order to achieve greater scope should not be taken to imply any disagreement with more tightly focused theories and models that offer higher degrees of resolution.

Differences between the purposes of the present model and those of other efforts also lead us to ignore certain generally accepted distinctions. For example, the present model treats all information-processingsteps as if they were equivalent even when we know that they involve different cognitive operations (e.g., mental rotation vs. visual search). Such distinctions, though clearly of fundamental importance for the purpose of understanding how cognitive tasks are accomplished, may be less important for purposes of understanding how individuals differ in the efficiency with which they accomplish these tasks.

Some of the simplifying assumptions made to facilitate the mathematical development of the model will be relaxed in subsequent computer simulations. For example, initially we will assume the existence of a "generic" computational step, and that differences in the RTs for different tasks or conditions reflect differences in the number of computational steps involved. In simulations, these assumptions will be relaxed so that the different steps involved in a given task may have different durations and so that the correlations between steps from the same task may be higher than those between steps from different tasks, reflecting possible task-specific abilities. Through the use of such simulations, and more importantly, by comparing the model's predictions to experimental data, we will attempt to show that, despite its deliberate simplification of the problem, our model is well suited to the task at hand: explaining the linkage between a general speed factor and the orderly increase in diversity of speeded performance with task difficulty.

\section{THEORETICAL DEVELOPMENT Preliminary Considerations}

As Cerella (1990) pointed out, orderly relations such as those shown in Figures 1 and 2 imply correspondence between the processing of the individuals involved. That is, such relations suggest that all (or most) individuals performing the tasks in question are processing the same information in the same way. Thus, the major determinant of the size of individual differences appears to be quantitative rather than qualitative and involves something that all the tasks have in common, albeit something that some tasks (i.e., those associated with longer RTs) have more of than others.

More specifically, an orderly relation between performances implies that these performances are functions of a third variable (Cerella, 1994; Dunn \& Kirsner, 1988; Hale 


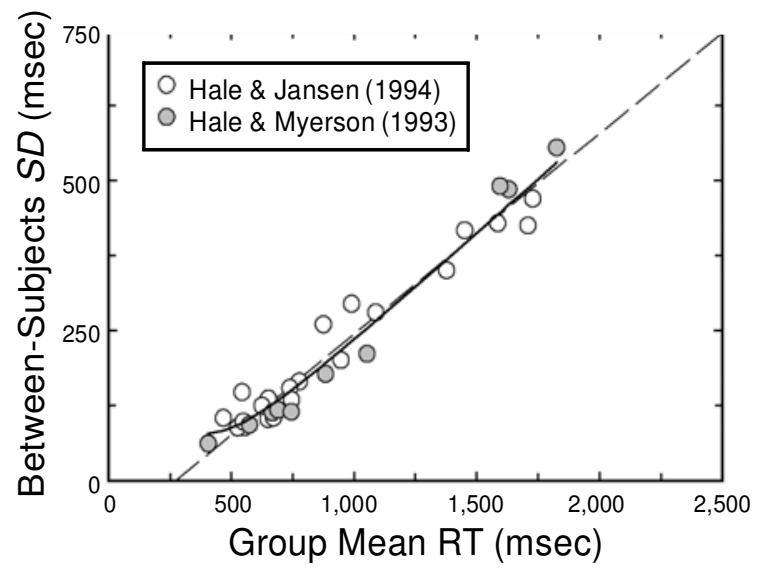

Figure 5. Between-subjects standard deviation $(S D)$ plotted as a function of group mean response time (RT). As in Figure 1, the data are taken from Hale and Jansen (1994) and Hale and Myerson (1993). Each circle represents the $S D$ and mean of the RTs for one experimental condition of one study, and the dashed regression line represents the fit to all of the data points. The solid curve represents the prediction of the difference engine model (Equation 7).

et al., 1987). Further understanding of this implication follows from an extension of ideas originally suggested by Dunn and Kirsner (1988) and extended to age differences in RTs by Cerella (1994). Dunn and Kirsner applied the chain rule, familiar to calculus students, to all cases of orderly relations between two types of performance. Although the chain rule is relevant in all such cases, it has special significance for the specific case of approximately linear relations observed here. In this case, the linear relation implies that the performances of fast and slow individuals are isomorphic functions of a third variable such that the effect of changes in that third variable on the performance of different individuals differs only proportionally. In addition, the fact that performance differences appear to be relatively independent of the type of processing involved, but increase with the average time required for task performance, suggests that this third variable may be characterized as a measure of the amount of processing required by a task. This insight, taken together with evidence for a general speed factor (e.g., Hale \& Jansen, 1994; Vernon \& Jensen, 1984; Vernon, Nador, \& Kantor, 1985; Zheng et al., 2000), provides the basis for our model of group and individual differences.

Diversity in performance is typically measured as the $S D$ for the group (rather than as the difference between fast and slow subgroups), and statistical theory typically treats variability in this form (and as the variance, or $S D$ squared). Therefore, we have recast the data represented in Figure 1 in these terms. As may be seen in Figure 5, the diversity of individual performances, measured as the group (or between-subjects) $S D$, increases with the amount of processing required in a task condition.

The fact that variability in RT tends to increase with the mean may seem unremarkable. It should be noted, how- ever, that in Figure 5 variability does not merely increase with the mean, but rather increases in a very orderly and highly specific manner. The approximately linear relation between mean and $S D$ accounts for more than $95 \%$ of the variance. Importantly, both the form and the values of the parameters appear to be robust characteristics of RT data. A single regression line describes the data from two different studies (Hale \& Jansen, 1994; Hale \& Myerson, 1993). In addition, linear relations between $S D$ and mean RT with very similar parameters (slopes of 0.3 to 0.4 and negative intercepts) were reported in a previous metaanalysis comparing the diversity of speed of performance in young and older adults (Hale et al., 1988). Even in the meta-analysis, where the data came from many different samples, the linear relation between $S D$ and mean RT accounted for more than $85 \%$ of the variance. Thus, the observed approximately linear relationship is not peculiar to data from one study, one laboratory, or even one age group.

Moreover, the form of the relation between the mean and $S D$ (or variance) can provide important information about the nature of the underlying process. How this applies to the present problem may be seen by considering a fundamental fact from elementary statistical theory. Specifically, the variance (VAR) of the sum of $n$ measures with variances $\sigma_{1}^{2}, \sigma_{2}^{2}, \ldots \sigma_{n}^{2}$ is given by the equation

$$
\begin{aligned}
\operatorname{VAR}= & \sigma_{1}^{2}+\sigma_{2}^{2}+\ldots+\sigma_{n}^{2}+2 r_{12} \sigma_{1} \sigma_{2} \\
& +2 r_{13} \sigma_{1} \sigma_{3}+\ldots+2 r_{n, n-1} \sigma_{n} \sigma_{n-1} .
\end{aligned}
$$

In the simple case where the means for the different measures are all equal, all of the variances are equal, and all of the correlations between the measures are equal, then

$$
\mathrm{VAR}=n \sigma^{2}+r n(n-1) \sigma^{2} .
$$

(We do not suggest that these simple assumptions are correct. Rather, we wish to see what insights can be gained from considering mathematically tractable cases before considering cases of sufficient complexity to require computer simulations.)

Inspection of Equation 2 reveals that if the measures are uncorrelated (i.e., $r=0$ ), then $\mathrm{VAR}=n \sigma^{2}$ and $S D=\sigma n^{1 / 2}$. To apply this to RTs, we assume that each measure represents the time required to execute a single computation, or one processing step. Thus, the mean RT for a given task condition will be proportional to the number of processing steps; that is, $\mathrm{RT}=\alpha n$, where $\alpha$ is the average duration for a single step. Substituting for $n$ in the preceding equation yields

$$
S D=\sigma(\mathrm{RT} / \alpha)^{1 / 2},
$$

where $\sigma$ is the $S D$ of step durations across individuals. Equation 3 implies that if the durations of the steps are uncorrelated, then the relation between the mean RT for a task condition and the $S D$ of the RTs for that condition will not be linear. Rather, it will be negatively accelerated because $S D$ is proportional to the square root of the mean RT. In contrast, if the steps are perfectly correlated (i.e., $r=1.0$, so that the same individuals who are the fastest at 


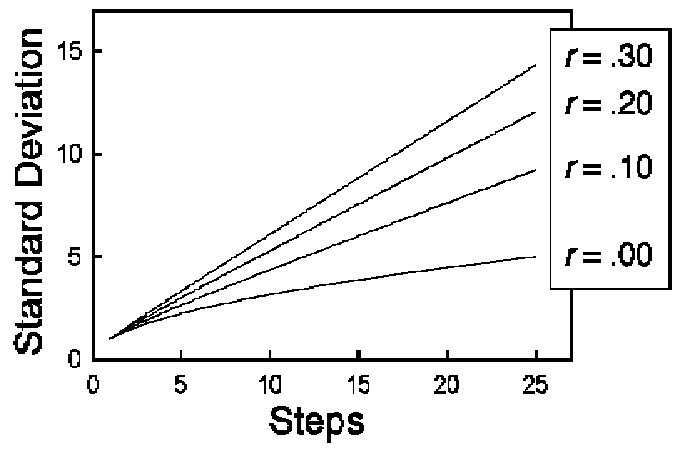

Figure 6. Standard deviation $(S D)$ of the sum plotted as a function of the number of steps being summed. Each curve represents the relation between $S D$ and number of steps given a particular correlation between the different measures. Calculations are based on Equation 5, and the correlation used to calculate each curve is given directly to the right of the curve.

one step will be the fastest at all other steps), then VAR = $n^{2} \sigma^{2}$. Substituting RT/ $\alpha$ for $n$ and taking the square roots of both sides of the equation yields

$$
S D=\sigma \mathrm{RT} / \alpha .
$$

Equation 4 reveals that if the durations of the steps are perfectly correlated, then the relation between mean RT and $S D$ will be linear. In fact, the $S D$ will be directly proportional to the mean.

How correlated do they have to be to yield an approximately linear relation between RT and $S D$ ? To answer this question, we may return to Equation 2 and, taking the square roots of both sides and rearranging, observe that for any $r$,

$$
S D=\sigma[n+n(n-1) r]^{1 / 2} .
$$

This equation may be used to assess the effect of the correlation between step durations on the relation between the number of processing steps and the $S D$, and thus on the relation between the number of steps and the $S D$ of RTs for a specific task condition.

Figure 6 illustrates what happens to the $S D$ of the sum of processing step durations as the number of steps and the magnitude of the correlation between them are varied. Although the figure depicts the simple case where the steps are all equally correlated and also have equal means and variances, the principle represented here undoubtedly holds as long as these assumptions are at least approximately true. That is, as may be seen in Figure 6 (which for simplicity also assumes unit variance), relatively low correlations between processing steps may serve to linearize the relationship between the number of steps and the $S D$.

As the correlation increases, so does the slope, whereas the intercept decreases. Although at higher correlations the $S D$ is approximately proportional to the number of steps, the general expectation based on Equation 5 is that intercepts will tend to be positive. Thus, as a descriptive model of RT data, Equation 5 suffers from an important limitation. Although it may explain why the relation be- tween $S D$ and RT is linear, it fails to predict the negative intercept clearly apparent in the present data (Figure 5) as well as in previous meta-analyses (e.g., Hale et al., 1988). As will be seen in the following section, this limitation is easily corrected, yielding a model that is more in line with intuition as well as providing a better description of the data.

\section{THEORETICAL DEVELOPMENT The Difference Engine}

\section{A Two-Compartment Model of Within-Groups Variability}

To understand how the observed negative intercept may be accommodated within the present approach, recall that Equation 5 assumes that any processing step is interchangeable with any other step of the same task (at least for the purpose of predicting individual differences). From the usual cognitive perspective, steps are obviously not interchangeable because they involve different computations or processing operations. In the present usage, however, steps are merely arbitrary measures of the amount of processing. Such generic steps serve the mathematically useful function of dividing what may, for other purposes, be thought of as a continuous flow (McClelland, 1979) into discrete units in the same way that a length may be divided into feet or, reflecting the arbitrary nature of the division, into meters.

The negative intercept demonstrates a limit to the usefulness of the mathematical fiction of generic, completely interchangeable steps, and accordingly, the strongest version of this fiction will be abandoned here in favor of a distinction similar to the familiar one between sensorimotor (or peripheral) and cognitive (or central) processing (e.g., Cerella, 1985). We assume a two-compartment model in which the duration of the response portion of a task is relatively uncorrelated with the duration of the central portion of that task.

Evidence consistent with this assumption is presented in Table 1, which reports the mean task RTs and intercorrelations from Hale and Myerson (1993). Note that the highest correlations are between the two easiest tasks (choice RT and letter classification) and between the two hardest tasks (visual search and abstract matching). This pattern presumably reflects the fact that sensorimotor speed plays a large role in easier tasks but makes a smaller contribution in more difficult tasks, whereas cognitive speed plays a large role in more difficult tasks but a smaller role in easier tasks. Consistent with previous studies, principal components analysis revealed that all tasks loaded heavily on the first principal component, which explained $75 \%$ of the variance. However, the second, bipolar, component explained an additional $12 \%$ of the variance. When varimax rotation was used to eliminate negative loadings and resolve a simple two-factor structure, the more difficult tasks (i.e., visual search and abstract matching) loaded highly on one factor (which may be interpreted as cognitive speed), whereas the easier tasks (i.e., choice RT and letter classification) loaded highly on the other factor (which may be interpreted as sensorimotor speed). 
Table 1

Mean Response Times (RTs) and Standard Deviations (SDs) in Seconds, Intertask Correlations, and Loadings on the First and Second Principal Components and First and Second Rotated Factors Extracted From the Hale and Myerson (1993) Data

\begin{tabular}{|c|c|c|c|c|c|c|c|c|c|}
\hline \multirow[b]{2}{*}{ Tasks } & \multirow[b]{2}{*}{$M \mathrm{RT}$} & \multirow[b]{2}{*}{$S D$} & \multicolumn{3}{|c|}{ Intercorrelations } & \multicolumn{2}{|c|}{ Unrotated } & \multicolumn{2}{|c|}{ Rotated } \\
\hline & & & CRT & LTR & SRC & $\mathrm{PC} 1$ & $\mathrm{PC} 2$ & RF1 & RF2 \\
\hline CRT & 0.405 & 0.061 & & & & .833 & -.470 & .292 & .911 \\
\hline LTR & 0.644 & 0.103 & .701 & & & .880 & -.197 & .512 & .743 \\
\hline SRC & 1.079 & 0.233 & .583 & .657 & & .863 & .321 & .879 & .321 \\
\hline MAT & 1.711 & 0.511 & .608 & .679 & .740 & .878 & .287 & .840 & .385 \\
\hline
\end{tabular}

Note-CRT, choice reaction time; LTR, letter classification; SRC, visual search; MAT, abstract matching tasks; PC1 and PC2, loadings on the first and second principal components; RF1 and RF2, loadings on the first and second rotated factors.

Similar patterns are seen in the correlations reported by Zheng et al. (2000) and also in the Hale and Jansen (1994) data. In the latter study, for example, the two easiest tasks (i.e., line-length discrimination and choice RT) had a correlation of .614, and the two most difficult tasks (i.e., abstract matching and mental paper folding) had a correlation of .646, whereas the correlation between the easiest and hardest tasks was .397. The same pattern was also seen in the correlations reported by Zheng et al. In both of these studies, the second principal component was bipolar, with loadings that covaried with task difficulty, and varimax rotation yielded one factor that may be interpreted as cognitive speed, for which the loadings were positively correlated with RT, and another factor that may be interpreted as sensorimotor speed, for which the loadings were negatively correlated with RT. Moreover, for all three studies, the correlations of both the cognitive and sensorimotor speed factors with task mean RT were .75 or stronger. With respect to the Hale and Jansen study, for example, the loading of the easiest task (line-length discrimination) on the sensorimotor speed factor was .906, whereas its loading on the cognitive speed factor was .109. In contrast, the loadings of the most difficult task (mental paper folding) on the sensorimotor and cognitive speed factors were .265 and .844 , respectively.

Although the assumption of complete independencebetween the cognitive and response components of RT is extreme, it greatly simplifies the mathematical development. We will continue, however, to assume that within the cognitive component, the durations of all processing steps are correlated, and equivalently so. Thus, the model developed in the previous section continues to apply to all of the cognitive processing that goes into RTs. The present model, which we have termed the difference engine, may be summarized as follows:

1. Cognitive task architecture. It is assumed that tasks and conditions that differ in difficulty also differ in the amount of cognitive processing (i.e., in the number of elementary computations or processing steps) that is required in order for an individual to make an accurate decision.

2. Cognitive step durations and correlations. For any individual performing any task, the durations of the steps that make up that task are correlated, and these correla- tions are assumed, on average, to be the same for all individuals and all tasks. This assumption provides the basis for the mathematical modeling that follows. Our computer simulations incorporate an additional assumption. More specifically, the correlations between steps of the same task are assumed to be higher than are those between steps of different tasks. Additionally, the within-tasks step duration correlations are assumed, on average, to be equivalent for all tasks, as are the lower, between-tasks step duration correlations.

3. Response step durations and correlations. The time it takes to report a decision by selecting and executing a minimal motor response (e.g., pushing a button) is assumed to vary across individuals and to be independent of the time they require to execute elementary cognitive processing steps or to reach a correct decision (i.e., their total cognitive processing times).

Before proceeding, we should note that we do not assert that all experimental tasks have a serial architecture like that assumed here, although many may. One could obviously find or devise tasks that do not satisfy this constraint, and our model would not apply to such tasks. Similarly, we do not assert that the time it takes an individual to report a decision is the same on all cognitive tasks. Rather, we would note that if one wants to study or manipulate processing time, it makes sense to keep the response requirement minimal as well as to hold it constant, as in the present model.

The uncorrelated response component may be incorporated into the model as follows. According to Equation 1, if there are only two, uncorrelated, items, VAR $=\sigma_{1}^{2}+$ $\sigma_{2}^{2}$. In the present application, $\sigma_{1}^{2}$ represents the variance of the duration of the response portion of a speeded task and $\sigma_{2}^{2}$ represents the variance of the duration of the cognitive portion. As just noted, the cognitive portion consists of a number of correlated processing steps, and therefore Equation 2 may be substituted for $\sigma_{2}^{2}$, yielding

$$
\mathrm{VAR}=\sigma_{r}^{2}+n \sigma_{c}^{2}+n(n-1) r \sigma_{c}^{2},
$$

where $\sigma_{r}^{2}$ is the variance for the response component and $\sigma_{c}^{2}$ is the variance for a single cognitive processing step.

In the present two-compartment model, the amount of cognitive processing time (i.e., the mean RT minus the duration of the response component, $t_{r}$ ) is proportional to the number of processing steps (i.e., RT $-t_{r}=\alpha n$, where $\alpha$ is 
the mean processing step duration). Substituting $\left(\mathrm{RT}-t_{r}\right) / \alpha$ for $n$ and rearranging yields

$$
\begin{aligned}
\mathrm{VAR}= & \sigma_{r}^{2}+\left[(1-r) \sigma_{c}^{2}\right]\left(\mathrm{RT}-t_{r}\right) / \alpha \\
& +r \sigma_{c}^{2}\left[\left(\mathrm{RT}-t_{r}\right) / \alpha\right]^{2},
\end{aligned}
$$

which is a second-order polynomial (i.e., $\mathrm{VAR}=b_{0}+$ $b_{1} \mathrm{RT}+b_{2} \mathrm{RT}^{2}$ ). Therefore, the $S D$ of the RTs is simply the square root of a second-order polynomial, shown as a solid curved line in Figure 5.

Although the fit here is only slightly better than that provided by the regression line, the theoretical equation (i.e., the square root of Equation 7) captures what turns out to be a reliable initial positive acceleration. According to the two-compartment model, this acceleration reflects the fact that as RTs get longer, the variance associated with the correlated steps of their cognitive component represents an increasingly larger proportion of the total variance. Unfortunately, although curve fitting provides estimates of the polynomial coefficients, these estimates are difficult to interpret because these coefficients represent composites of the parameters of Equation 7. Moreover, although the parameters of Equation 7 have specific interpretations, these parameters are highly interdependent and therefore difficult to estimate. Thus, although the very good fit and intuitively reasonable form of our theoretical equation provide support for the logic underlying this equation, an alternative approach will be needed for some purposes.

One such approach capitalizes on the fact that as RT grows larger, the rightmost expression (i.e., the one containing the quadratic term) in Equation 7 comes to dominate, and the variance is increasingly well approximated by

$$
\mathrm{VAR}=r \sigma_{c}^{2}\left[\left(\mathrm{RT}-t_{r}\right) / \alpha\right]^{2} .
$$

Taking the square root of both sides yields

$$
S D=\left(r-\sigma_{c} / \alpha\right)\left(\mathrm{RT}-t_{r}\right) .
$$

This equation provides a basis for understanding the parameters of the approximately linear relation between $S D$ and mean RT. The slope, $m=r-\sigma_{c} / \alpha$, reflects both the correlations among processing step durations within a task and the coefficient of variation for cognitive processing (i.e., the between-subjects $S D$ of processing step durations divided by the mean duration). The $y$-intercept is simply $-m t_{r}$, and setting $S D$ equal to zero and solving for RT reveals that the $x$-intercept is equal to the duration of the response component, $t_{r}$. As may be seen in Figure 5, the observed $x$-intercept of approximately $300 \mathrm{msec}$ is in keeping with values for simple and choice RTs to highly discriminable stimuli reported in the literature (for a review, see Teichner \& Krebs, 1974) and is therefore consistent with the two-compartment assumption of our difference engine model.

\section{Extending the Model to Performance of Individuals and Subgroups}

To this point, the focus has been on the distribution of speeded performance across individuals and how this distribution changes with task difficulty (i.e., with variations in the number of processing steps). In the present section, we focus on the performance of specific individuals or of subgroups of individuals of similar ability and follow their performances from task to task. In subsequent sections, we will show that the difference engine may be extended further, enabling one not only to follow individuals from task to task but also in principle to follow their performance as they change with age and health status throughout the adult life span.

We begin by considering the difference engine's predictions regarding the interaction of individual differences
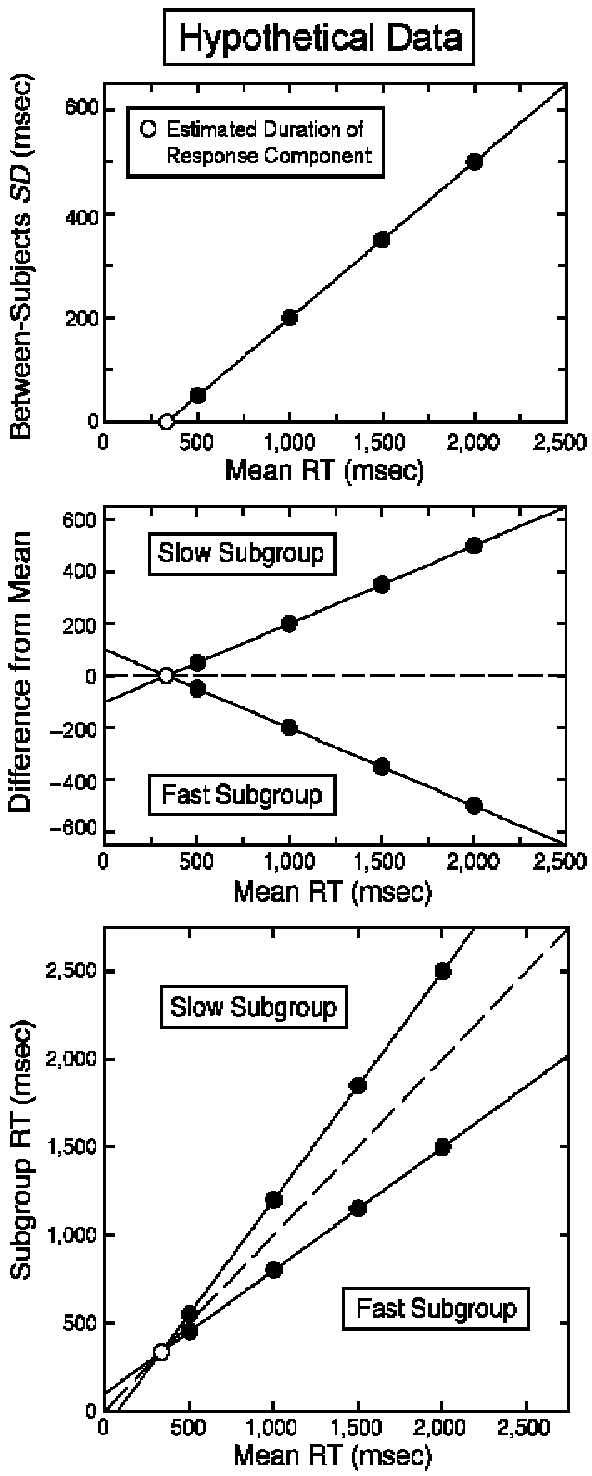

Figure 7. Graphical summary of the difference engine's predictions regarding individual differences within a group. The top panel illustrates the relation between standard deviation $(S D)$ and group mean response time $(\mathrm{RT})$, the middle panel illustrates the consequences of this relation for the RTs of subgroups $1 S D$ faster and $1 S D$ slower than average, and the bottom panel depicts the consequences for the relations between the RTs of these subgroups and the group mean RT. 
with task difficulty. The logic behind these predictions is straightforward and is summarized graphically in Figure 7. All of the hypothetical data depicted in the figure are calculated assuming fast and slow subgroups whose processing times are, respectively, $1 S D$ shorter and $1 S D$ longer than average, and also assuming that $S D=0.3 \mathrm{RT}-0.1$ (consistent with both Equation 9 and the empirical data presented previously in Figure 5).

As shown in the upper panel, the diversity of individual performance (indexed by the between-subjects $S D$ ) in- creases linearly with task difficulty (indexed by the mean RT). Because the speed with which individuals execute different processing steps is correlated, both within and between tasks, the same individuals tend to have long (or short) RTs on all cognitive tasks. The consequences of this tendency for the RTs of fast and slow subgroups may be seen in the middle panel of Figure 7. Specifically, the pattern of increasing diversity in the upper panel results in a similar linear increase in the difference between slow and average RTs. The difference between fast and average RTs

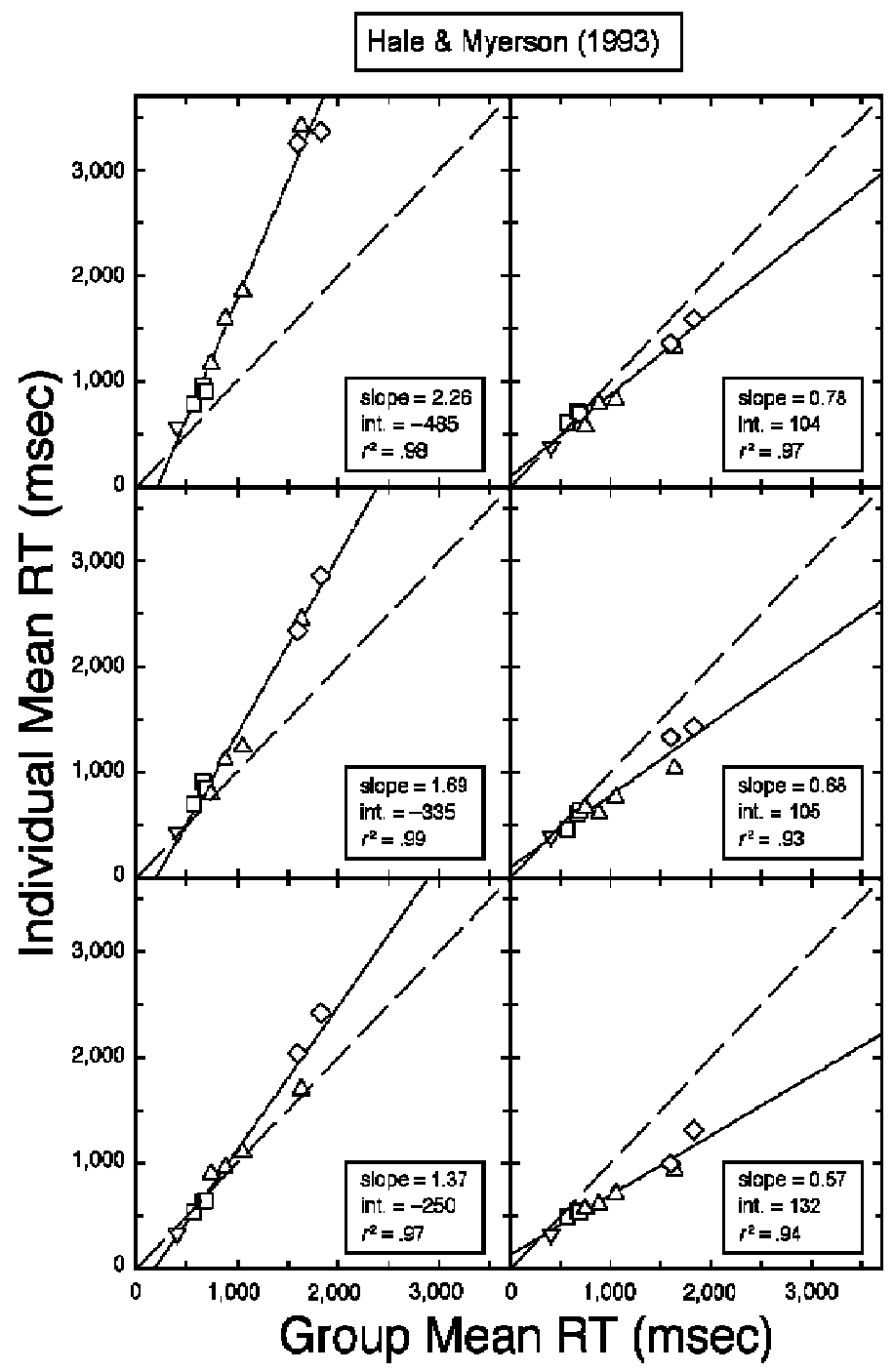

Figure 8. Response times (RTs) for representative individuals from Hale and Myerson (1993) plotted as a function of group mean RT. Each data point represents performance in one experimental condition. In order of increasing task difficulty, the tasks were as follows: inverted triangles, choice reaction time; squares, letter classification; upright triangles, visual search; diamonds, abstract matching. Data from the slowest and the fastest individuals are shown (upper left panel and lower right panel), as well as data from individuals at the 10 th, 33rd, $67 \mathrm{th}$, and 90 th percentiles (middle left, lower left, upper right, and middle right panels, respectively). If an individual's RT for a particular task condition did not differ from the corresponding group mean RT, the data point for that condition would fall along the dashed diagonal line. 
also increases linearly with task difficulty, although in this case the difference is negative.

Finally, converting the differences shown in the middle panel into mean RTs for each subgroup has the effect of rotating the graph so as to produce the graph depicted in the lower panel. Thus, as may be seen, combining a general cognitive speed factor with an approximately linear relation between $S D$ and mean RT leads logically to the correct prediction of linear relations between the RTs of subgroups of fast and slow individuals and the average RTs for the group as a whole (Figure 1).

Although plots of difference scores (e.g., the middle panel of Figure 7) provide an intuitively accessible way to visualize individual differences, for purposes of parameter estimation it is preferable to avoid difference scores and conduct regressions using RTs in the manner diagrammed in the bottom panel. Interpretation of the parameters of such regressions is straightforward. Consider an individual $(i)$ who is consistently slower than the mean by $1.0 S D$ (i.e., $\mathrm{RT}_{i}=\mathrm{RT}_{\mathrm{avg}}+S D$ ), and recall that $S D$ is approximately equal to $m \mathrm{RT}_{\text {avg }}-m t_{r}$ (Equation 9, the linear approximation of the relation between $S D$ and mean RT). It follows directly that the RTs of such an individual will be approximately equal to $(1+m) \mathrm{RT}_{\text {avg }}-m t_{r}$. More generally, for any individual,

$$
\mathrm{RT}_{i}=\left(1+z_{i} m\right) \mathrm{RT}_{\mathrm{avg}}-z_{i} m t_{r},
$$

where $z_{i}$ is a measure of individual processing speed in $S D$ units and $m=r-\sigma_{c} / \alpha$ (see Equation 9). This equation provides a guide to interpretation of the regression parameters. Because fast individualshave negative $z$ scores, they will have slopes less than 1.0 and positive $y$-intercepts. Slow individuals, who have positive $z$ scores, will have slopes greater than 1.0 and negative $y$-intercepts. Moreover, individual regression lines will tend to cross the diagonal (i.e., $\mathrm{RT}_{i}=\mathrm{RT}_{\mathrm{avg}}$ ) at a point where $\mathrm{RT}$ s are minimal (i.e., when the task is simple and the RT consists largely of sensorimotor time), although prediction at the individuallevel is complicated by both the nonlinearity of the relation between $S D$ and RT and the fact that sensorimotor time varies between individuals.

The results of analyses of the individual data from the 65 subjects in the Hale and Myerson (1993) study are consistent with predictions based on Equation 10. Strong linear relations were observed between individuals' condition mean RTs and group condition means as evidenced by very high individual $r^{2} \mathrm{~S}$ (median $r^{2}=.950$ ). Individual examples (fastest and slowest as well as those at the 10th, 33rd, 67th, and 90th percentiles) are presented in Figure 8. As predicted, regressions for slow individuals tended to have negative intercepts whereas those for fast individuals tended to have positive intercepts (for further examples of individual data, see Hale \& Jansen, 1994, and Zheng et al., 2000). In fact, 20 out of the 21 subjects with regression slopes greater than 1.05 had negative intercepts, and 34 of the 35 subjects with slopes less than 0.95 had positive intercepts. Moreover, individual regression lines tended to cross the diagonal in the region where expected (median crossing point at RT $=439 \mathrm{msec}$ ).
The use of Equations 9 and 10 for predicting the slopes of the linear relation between an individual or subgroup's RTs and the group mean RT may be illustrated using the data from the fast and slow subgroups in the Hale and Myerson (1993) study. For the Hale and Myerson data, linear regression of the group $S D$ on RT yielded an estimate of $m=0.377$ (Equation 9). According to Equation 10, the formula for the slope $(s)$ for an individual or subgroup is given by $s=1+z m$. For the fast subgroup, the grand mean $z$ (i.e., the average of the mean $z$ scores for the 16 members of the subgroup) was 1.11. Thus, substituting for $m$ in preceding formula, the predicted slope for this subgroup (i.e., 1 plus 1.11 times 0.377 ) equals 1.42 , very close to the observed slope of 1.45. For the fast subgroup, the grand mean $z$ was -1.01 , and thus the predicted slope ( 1 minus 1.01 times 0.377 ) equals 0.62 , again very close to the observed slope of 0.65 .

The same method for predicting slopes was also applied to the data from each of the 65 subjects in Hale and Myerson (1993). With no free parameters, the formula $s_{i}=1$ $+0.377 z_{i}$, where $s_{i}$ and $z_{i}$ represent the slope and mean $\mathrm{z}$ for the $i$ th individual, accounted for nearly three fourths of the variance in the observed slopes $\left(r^{2}=.726\right)$. Moreover, when the observed slopes were regressed on the predicted slopes, the slope of the regression did not differ significantly from 1.0 and the intercept did not differ significantly from 0.0 , suggesting that our prediction formula, based on Equation 10, was an unbiased predictor of individual regression lines.

What is important about the present effort is that it offers a relatively simple theoretical mechanism to predict and explain a whole set of empirical relationships. The approximately linear relation between $S D$ and group mean $\mathrm{RT}$ is explained as the consequence of correlations between processing step durations-faster individuals taking generally less time on all steps of a cognitive task and slower individuals taking generally more time. Taken together with the strong general speed factor revealed by principal component analysis, the relation between $S D$ and mean RT implies that the difference between fast and slow individuals will increase with the task mean RT, something not predicted by a general speed factor alone. This increase in individual differences is reflected in the specific form of the linear relation between individual condition mean RTs and group condition mean RTs. Thus, the difference engine appears to provide an integrated account of various aspects of RT data that are usually treated as separate phenomena.

\section{Computer Simulations of the Difference Engine}

The mathematical development presented in the previous two sections necessitated many simplifying assumptions. In order to assess the consequences of relaxing some of those assumptions and to set the stage for modeling performance in different groups, we next proceeded to develop computer simulations that embodied the principles of the difference engine. As before, RTs were assumed to consist of two components, one sensorimotor and the other cognitive. The cognitive component was assumed 


\section{Difference Engine Simulations}

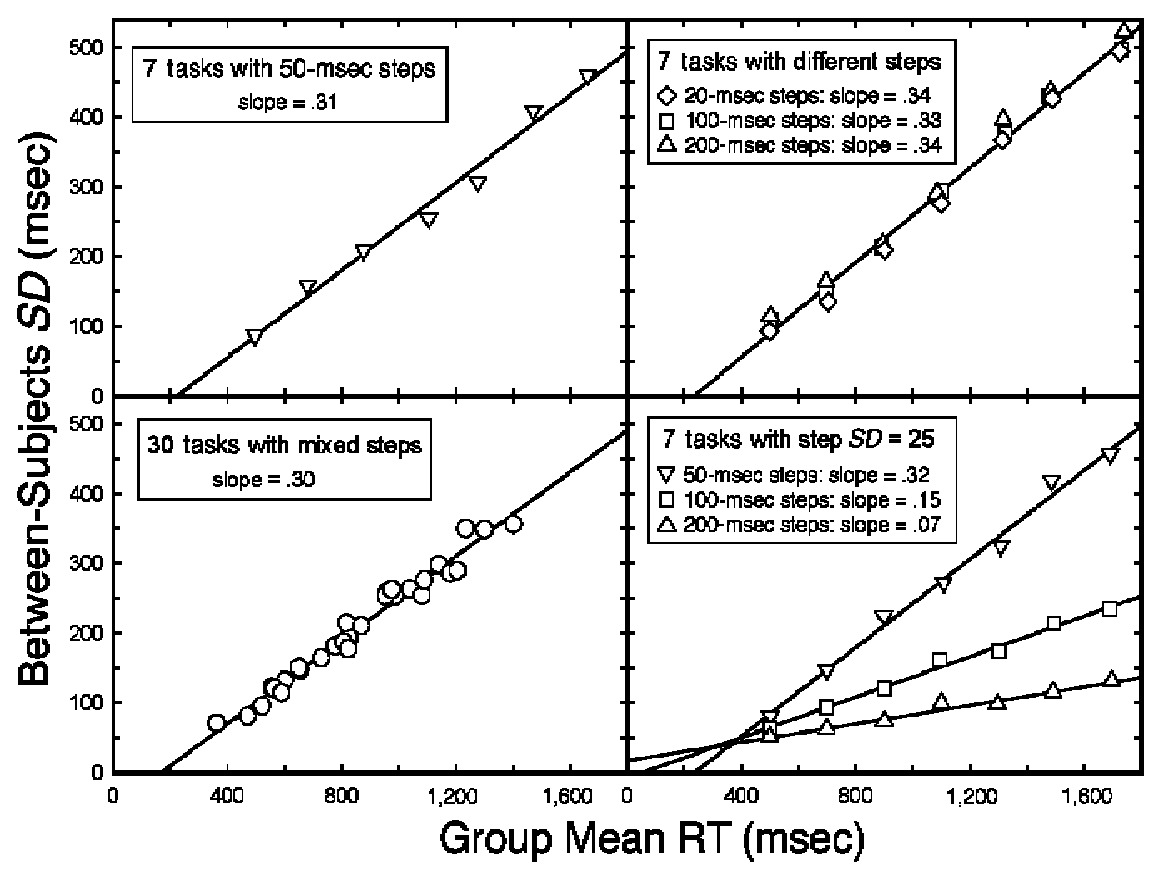

Figure 9. Between-subjects standard deviation (SD) for computer-simulated data plotted as a function of group mean response time (RT). The upper left panel presents simulation results for a mean step duration of $50 \mathrm{msec}$, and the upper right panel presents results for mean step durations of 20, 100, and $200 \mathrm{msec}$. The lower left panel presents simulation results for tasks consisting of a mixture of step durations, and the lower right panel presents results for simulations in which the mean step duration was varied from 50 to $200 \mathrm{msec}$ but the $S D$ was held constant at $25 \mathrm{msec}$ (see text for details). For all step durations but those shown in the lower right panel, the coefficient of variation was .5. Note that to facilitate comparisons, we made the ratio of the scale of the $x$-axis to the scale of the $y$-axis the same as in Figure 5.

to represent the sum of the duration of individual processing steps, and these step durations were assumed to be correlated both within and between tasks. The sensorimotor component was assumed to be $300 \mathrm{msec}$ on average, with a $S D$ of $50 \mathrm{msec}$ across individuals. Processing step durations were assumed to be $50 \mathrm{msec}$ with a $S D$ of $25 \mathrm{msec}$ across individuals and tasks, and uncorrelated with individuals' sensorimotor component.

Across tasks, the correlation between an individual's processing steps was set at .35 , on average, whereas within tasks the correlation was set at .50. (These assumptions only provided target values, both because of random variation per se and because such variation occasionally, albeit rarely, produced negative step durations that were automatically replaced with zeros.) Correlations were produced by calculating each cognitive step duration as the sum of three random variables. More specifically, each simulated subject was randomly assigned a general cognitive speed value as well as an independently chosen task-specific speed for each task. Then each step duration was assumed to be the sum of these first two random variables plus a third, independent, value. This third random variable may be thought of as a combination of intrinsic variation and measurement error. RTs were calculated as the sum of the step durations plus a fourth random variable that represented subjects' sensorimotor speed. Correlations between task steps reflected the shared general and task-specific variables, whereas correlations between RTs for different tasks reflected the general cognitivespeed variable and the sensorimotor speed variable.

Consider a typical simulation with parameters chosen to approximate the tasks used by Hale and Jansen (1994) and Hale and Myerson (1993). For each of 100 simulated subjects, we generated RTs for seven tasks consisting of 4 , $8,12,16,20,24$, and 28 processing steps. The mean RTs for these tasks ranged from approximately $500 \mathrm{msec}$ for the 4-step task (300 msec of sensorimotor time plus $200 \mathrm{msec}$ of information processing) to $1,700 \mathrm{msec}$ for the 28 -step task. Characteristics of the simulated steps (i.e., mean durations, $S D$ s, and intercorrelations) were reasonably close to target values, and the first principal component accounted for $69 \%$ of the variance. As may be seen in the upper left panel of Figure 9, the relationship of between-subjects $S D$ and task mean RT for this simula- 
tion closely approximated that seen in the actual data (see Figure 5).

Additional simulations were conducted varying the mean step duration while holding constant the target values for the step intercorrelations and the ratio of mean step duration to the step duration $S D$. Targets for task mean RTs were also held constant. For example, with 20-msec steps $(S D=10 \mathrm{msec})$ the easiest and hardest tasks consisted of 10 and 70 steps, respectively, whereas with $200-\mathrm{msec}$ steps $(S D=100 \mathrm{msec})$ the corresponding tasks consisted of 1 and 7 steps. Consistent with Equation 9, the relationship between $S D$ and mean RT generally was not affected by these manipulations. This may be seen in the upper right panel of Figure 9.

To examine the effect of mixing step durations, tasks were simulated using $3,4,5,6,7$, or 8 steps with RTs being made up of randomly selected mixtures of 20-, 50-, $100-$, and 200-msec step durations. There were 30 tasks in all, five for each number of steps. Target values for step duration, intercorrelations, and the ratio of mean step duration to the step duration $S D$ were the same as in previous simulations. As may be seen in the lower left panel of Figure 9, the relationship between $S D$ and mean RT for simulations in which the steps making up a task had different mean durations was similar to the relationship between $S D$ and mean RT for simulations in which the steps making up a task had the same duration.

These simulation results suggest that the characteristics of the difference engine are fairly robust as long as the intercorrelations between step durations and the ratio of mean step duration to the step duration $S D$ are not varied. Although we did not search the entire space for boundary conditions, we did use simulations to evaluate those constraints predicted by the model (e.g., Equation 9). For example, we examined the effect of increasing mean step durations without increasing their variability. As may be seen in the lower right-hand panel of Figure 9, increasing the mean step duration from 50 to 100 to $200 \mathrm{msec}$ while holding the $S D$ of step durations constant at $25 \mathrm{msec}$ had the predicted effects of decreasing the slope of the regression of between-subjects $S D$ on mean RT and increasing the intercept. As expected on the basis of Equation 9, similar effects were observed with different pairs of starting values. That is, increasing the mean step duration while holding variability constant resulted in regression lines with parameters that diverged systematically from those observed with real data. Also as expected on the basis of Equation 9, decreasing the correlations between step durations (with the target values for mean step duration and $S D$ held constant at 50 and $25 \mathrm{msec}$, respectively) had the effect of decreasing slopes and increasing intercepts.

We conducted one further test of the robustness of the difference engine model. Distributions of RTs across individuals typically are not normal (i.e., Gaussian), but instead are positively skewed. That is, the difference between the RTs of the slowest individuals and the group mean is much greater than the difference between the RTs of the fastest individuals and the group mean. Because the simulations just described assumed Gaussian distribu- tions, additional simulations were conducted to verify that the present results do not depend on this assumption. Accordingly, skewed distributions were created by taking the correlated step durations generated by our simulation program and raising them to the 1.5 power. In addition to skewing the distribution, this transformation also had the effect of increasing the mean, and therefore all cognitive step durations were decreased proportionally so that the mean was approximately $50 \mathrm{msec}$.

The RTs for seven tasks were generated by adding 4,8 , $12,16,20,24$, and 28 processing steps, as in our initial simulation, and the resulting task mean RTs ranged from approximately $500 \mathrm{msec}$ for the 4 -step task $(300 \mathrm{msec}$ of sensorimotor time plus $200 \mathrm{msec}$ of information processing) to approximately $1,700 \mathrm{msec}$ for the 28 -step task. With correlations between cognitive step durations as low as .30, the distribution of RTs for each task, measured across 100 simulated individuals, was positively skewed, and more importantly, as predicted by the difference engine, the relationship of between-subjects $S D$ to task mean RT was linear with a negative intercept. With lower correlations, the relation between $S D$ and mean RT was negatively accelerated, also as predicted. Furthermore, for correlations that produced linear relations between $S D$ and mean RT, the slope was an increasing function of the correlation between step durations, again as predicted. These simulation results obtained with skewed distributions of step durations parallel those obtained with our mathematical model and with computer simulations that assumed Gaussian distributions.

Taken together, the results of our simulations with both normal and skewed step durations help confirm that the behavior of the difference engine, although robust across certain manipulations, does depend on the relationships between certain parameters in the way predicted by the model (see also further simulation results presented in the next section). Importantly, simple assumptions about serial processing other than those incorporated in the difference engine, such as uncorrelated step durations or variability in step durations independent of the mean duration, did not result in simulations consistent with the patterns observed with real data.

\section{THE DIFFERENCE ENGINE AND COGNITIVE SLOWING}

\section{Extending the Model to Performance of Different Groups}

We are now ready to consider the role of individual characteristics other than ability (i.e., age and health status). Our basic assumptions regarding cognitive task architecture, step durations, and correlations between steps remain unchanged. That is, tasks and conditions that differ in difficulty are assumed to differ in the number of elementary processing steps required in order to make an accurate decision, and cognitive step durations are assumed to be correlated with each other but not with the duration of the response step (see Assumptions 1, 2, and 3 of the two-compartment model of within-groups variability). In 
extending the model to different groups, for simplicity's sake we assume that all individuals affected by the same age or health status are affected to the same degree on all speeded processing tasks, at least within broad cognitive domains (e.g., the verbal domain and the visuospatial domain). More specifically, the difference engine incorporates the following assumptions in addition to the three already described:

4. Cognitive step durations and correlations. It is assumed that aging and many health conditionsincrease the average time that each affected individual requires to complete one step of cognitive processing by some proportion, $s_{c}$, which is specific to the particular age or condition. As a consequence, the between-subjects $S D$ of step durations is increased by the same proportion so that the mean and $S D$ are $s_{c} \alpha$ and $s_{c} \sigma_{c}$, respectively. Other informationprocessing parameters of the difference engine (e.g., the magnitude of the intercorrelations) are unaffected by this increase in step durations.

5. Response step durations and correlations. It is assumed that aging and many health conditions may also increase the time it takes to report a decision. The proportion, $s_{r}$, by which this component of performance is increased is assumed to be specific to a particular age or condition and may differ from the proportion by which cognitive processing times are increased. Although the duration of cognitive and response steps may covary across groups, it is assumed that within a group the time it takes to select and execute a minimal motor response is independent of the duration of an elementary cognitive processing step.

It should be noted that the difference engine continues to assume correspondence; that is, regardless of age or health status, it is assumed that all (or most) individuals performing speeded cognitive tasks are processing the same information in the same way (Cerella, 1990). Thus, the major determinants of the size of individual and group differences are assumed to be quantitative. It may be possible to find or devise tasks that do not satisfy this constraint; our model would not apply to such tasks. We would point out, however, that in order to study group differences in processing speed, as opposed to group differences in strategy, it would make sense to focus on tasks in which there is correspondence in processing.

The preceding assumptions provide the basis for the mathematical modeling and computer simulations that follow. In order to make these efforts more concrete, we will consider age to be the paradigmatic individual characteristic and compare the performance of older adults against a young adult standard. Assuming that aging affects the duration of cognitive processing steps but not their intercorrelations, we may derive the relation between $S D$ and mean RT for an older adult group using the same logic as that used previously. To summarize, the difference engine predicts a relation between $S D$ and mean RT that is described by the square root of a second-order polynomial (Equation 7). Further, as the number of processing steps increases, this equation becomes increasingly well approximated by a linear function (Equation 9): $S D=$ $\left(r-\sigma_{c} / \alpha\right)\left(\mathrm{RT}-t_{r}\right)$.
In the present case, where there is both cognitive slowing (affecting the mean and $S D$ of cognitive step durations equally) and response slowing, this equation becomes

$$
S D=r-\left(s_{c} \sigma_{c} / s_{c} \alpha\right)\left(\mathrm{RT}-s_{r} t_{r}\right) .
$$
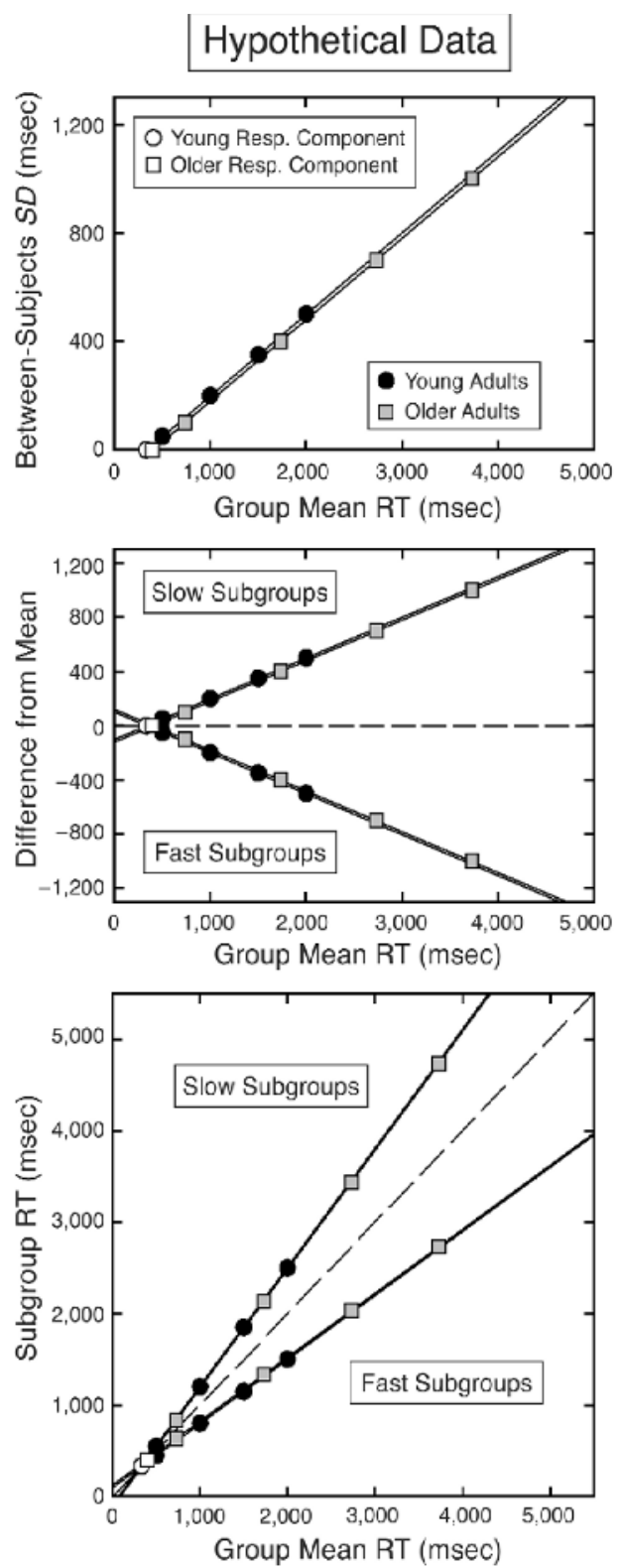

Figure 10. Graphical summary of the difference engine's predictions for individual differences within different age groups. The upper panel illustrates the relations between the standard deviation $(S D)$ and mean response time (RT) for groups of young and older adults. The middle panel illustrates the consequences of these relations for the RTs of young and older subgroups who are $1 S D$ faster and $1 S D$ slower than average for their age group. The lower panel depicts the further consequences for the relations between the RTs of these subgroups and the mean RTs of the corresponding age groups (i.e., fast and slow young as a function of average young performance and fast and slow older as a function of average older performance). 
It may be noted immediately that $s_{c}$, the cognitive slowing coefficient, cancels out. Thus, the slope parameter simplifies to $m=r-\sigma_{c} / \alpha$, which is the same as in Equation 9, and Equation 11 may be rewritten as

$$
S D=m \mathrm{RT}-m s_{r} t_{r}
$$

The strong and perhaps unexpected implication is that identical slopes are predicted for young and older groups. Setting $S D$ equal to zero and solving Equation 11 for RT reveals that the $x$-intercept for the older group is the young adult intercept multiplied by $s_{r}$, the coefficient that reflects the slowing of the response component with age. Because the degree of sensorimotor slowing in older adults is typically quite small (Cerella, 1985), the age difference in intercepts also will be quite small and, as a result, may not be statistically reliable.

Moreover, because the relationshipbetween $S D$ and mean $\mathrm{RT}$ is relatively unaffected by aging, the difference engine predicts that the form of the regression of individual and subgroup RTs on group mean RT will be the same for young and older adults. The relationshipsbetween these various predictions are summarized graphically in Figure 10, which adds a hypothetical older group to the young adult group previously depicted in Figure 7. For purposes of calculating these hypothetical data, we assumed that in older adults, information processing is slowed by a factor of 2.0, typical of nonverbal cognitive tasks (e.g., Lima, Hale, \& Myerson, 1991; Sliwinski \& Hall, 1998), whereas sensorimotor processing is slowed by a factor of only 1.15 (Cerella, 1985).

As may be seen in the upper panel of Figure 10, the diversity of individual performance (as indexed by the $S D$ for the group) increases linearly with task difficulty (as indexed by the mean RT) for both groups. According to the difference engine, the slope of the relation is identical for both young and older adult groups, but the $y$-intercept for the hypothetical older adult group is more negative, reflecting age differences in the response component. Response selection and execution are only slightly (i.e., approximately $15 \%$ ) slower in the older group, however, and therefore the difference between the lines for the two groups is barely discernible.

For both age groups, the speed with which individuals execute different processing steps is correlated, so that the same individuals tend to have relatively long (or short) RTs for their age on all tasks. Thus, the pattern of increasing diversity in the top panel leads to linear increases in the difference between average RTs, on the one hand, and the RTs of fast and slow subgroups, on the other hand (see the middle panel of Figure 10). Finally, the RTs for each subgroup are equal to the mean for their age group plus the difference from the mean. Converting the differences depicted in the middle panel into the subgroups' mean RTs produces the graph depicted in the lower panel, in which the difference between the regression lines for the fast older and fast young subgroups, which have the same slopes but differ slightly with respect to their intercepts, is hard to discern, and the same is true for the difference between the regression lines for the slow older and slow young subgroups.
Zheng et al. (2000) recently reported results consistent with the present model. The young adult and older adult groups in the Zheng et al. study consisted of 40 subjects each, all of whom were tested on seven visuospatial tasks: choice RT, disjunctive choice RT, colored shape discrimination, two-object same/different judgment, three-object same/different judgment, and two different types of abstract matching tasks. Zheng et al. reported that a general speed factor accounted for $77 \%$ and $70 \%$ of the variance in standard scores for the young and older groups, respectively. Moreover, the composition of the general speed factor was relatively age invariant, as implied by the difference engine's assumption that aging does not affect the intercorrelations between step durations. Specifically, there was a correlation of .99 between the observed factor scores for individualolder adults and the factor scores pre-
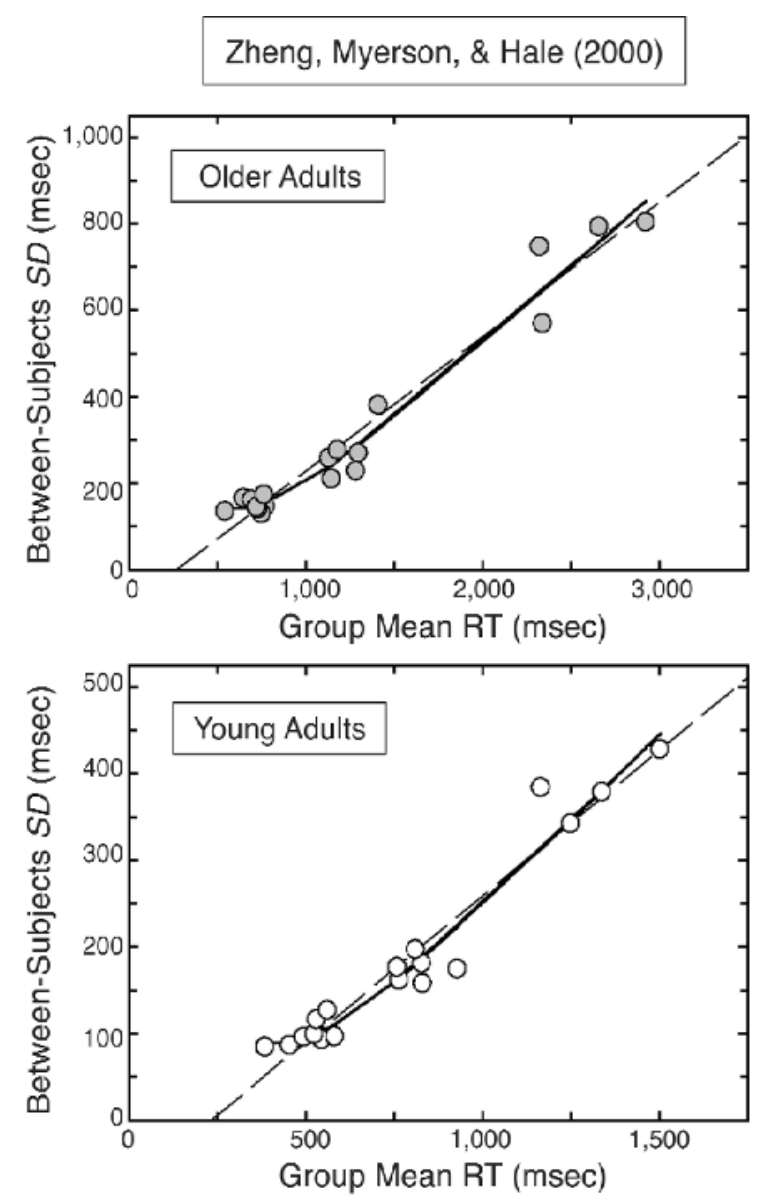

Figure 11. Between-subjects standard deviation $(S D)$ plotted as a function of group mean response time (RT). Data are taken from Zheng, Myerson, and Hale (2000). Each circle represents performance by one age group in one experimental condition. Data from older and young adults are presented in the upper and lower panels, respectively. Note that the scales in the two panels are different, but that the ratios of the scale of the $x$-axis to the scale of the $y$-axis are the same as in Figure 5. In each panel, the dashed line represents the linear regression equation and the solid curve represents the prediction of the difference engine (Equation 7). 
dicted on the basis of the loadings from the young adults' data. This finding suggests that the composition of the general speed factor in the two age groups was highly similar (Gorsuch, 1983). Furthermore, regression of the RTs of the fast young and fast older adults on the average RTs for their peers yielded virtually identical slopes and intercepts and accounted for more than $98 \%$ of the variance in both cases. Regression of the RTs of the slow young and slow older adults on the average RTs for their peers also yielded nearly equivalent slopes and intercepts, and again accounted for more than $98 \%$ of the variance in both cases.

According to the difference engine, the necessary theoretical linkage between the factor analytic findings and the regression analysis of fast and slow subgroups' RTs is provided by the effect of correlated step durations on the relation between $S D$ and mean RT. In order to test the model's predictions regarding the relation between $S D$ and mean RT in young and older adults, we reanalyzed the Zheng et al. (2000) data for each group separately. As may be seen in Figure 11, the data from both the young and older adult groups are well described by linear functions (Equations 9 and 11, respectively). Notably, the $x$-intercept estimate of the response component for the older group was $16 \%$ greater than that for the young group, very close to Cerella's (1985) meta-analytic estimate of $15 \%$ sensorimotor slowing. The slopes of the regression lines for the young and older groups (.311 and .337, respectively) are both very similar to those for the two large young adult samples (Hale \& Jansen, 1994; Hale \& Myerson, 1993) shown in Figure 3 as well as to those reported by Hale et al. (1988) in their meta-analysis of published studies from a number of different laboratories, further attesting to the reliability of this phenomenon.

Because the Zheng et al. (2000) study tested subjects on a number of fairly easy tasks that produced short RTs as well as on tasks that produced fairly long RTs, the data afford an opportunity to test for the positive acceleration in the relation between $S D$ and RT predicted by the difference engine. Both young and older adults showed significant positive acceleration (i.e., in each case, polynomial regression revealed the presence of a significant quadratic term), as predicted by Equation 7. Thus, these data support the difference engine's predictions regarding the form of the relation between $S D$ and mean RT as well as being consistent with the model's assumption that the major effect of aging on RTs results from simply increasing the durations of the steps required to perform informationprocessing tasks.

\section{Extending the Model \\ to Domain-Specific Slowing}

Lima et al. (1991) conducted a meta-analysis of studies of age-related cognitive slowing, and they reported that the degree of age-related slowing on visuospatial tasks was considerably greater than that on verbal tasks of approximately the same degree of difficulty, as indexed by the RTs of young adults. However, the studies analyzed by Lima et al. used either verbal or visuospatial tasks, but not both, so that the comparison of verbal and visuospatial slowing necessarily involved different samples, and the motor requirements were not always equivalent in different studies. In an experimental validation of the Lima et al. findings, Hale and Myerson (1996) tested the same 24 young and 24 older adults on both verbal and visuospatial tasks, all of which had equivalent response requirements (i.e., pressing the correct one of two response keys). There were four different verbal tasks (i.e., single lexical decision, double lexical decision, category membership, and synonym/antonym judgment) and four different visuospatial tasks (i.e., line-length discrimination, shape classification, visual search, and abstract matching).

Analyses indicated that the older adult group was more than $200 \%$ slower, on average, than the young adults at processing visuospatial information, whereas they were less than $50 \%$ slower than the young adults at processing verbal information. Taken together, the correspondence between these experimental results (Hale \& Myerson, 1996) and the meta-analytic results reported by Lima et al. (1991) attests to the robustness of the phenomenon (i.e., it is not specific to a particular sample or to one specific laboratory and its procedures). More specifically, the degree of age-related slowing (i.e., the value of $s_{c}$ according to the difference engine notation) is determined to a considerable extent by the domain (i.e., verbal or visuospatial), rather than by other specific task characteristics. Notably, the degree of age-related slowing is much greater in the visuospatial domain. This may be seen by comparing the upper and lower panels of Figure 4: The differences between the RTs of the three age groups on visuospatial tasks (upper panel) are much greater than the differences on verbal tasks (lower panel).

The finding of greater age-related slowing in the visuospatial than in the verbal domain observed at the group level also appears to be a reliable characteristic of age-related slowing at the individual level. When the RTs of individual subjects are regressed on the means for a young adult control group, the percentage with greater visuospatial than verbal slopes increases systematically with age from $50 \%$ of young adults to $98 \%$ of individuals more than 60 years of age (Lawrence et al., 1998). Because of the differential effects of age on visuospatial and verbal speed at both the individual and group levels, the two domains provide an important opportunity to test the present difference engine model.

The difference engine makes clear predictions for the two domains regarding both the regression of $S D$ on mean $\mathrm{RT}$ and the regression of the RTs of fast and slow subgroups on the mean RTs for their age group. That is, just as the slope $(m)$ of the regression of $S D$ on mean RT is predicted to be independent of age, so $m$ is predicted to be independent of domain. This is because, as already shown, the cognitive slowing coefficient cancels out of the equation for the regression of $S D$ on mean RT (Equation 12), and thus $m$ is independent of the degree of age-related cognitive slowing. The degree of age-related cognitive slowing changes from one domain to the other, but $m$ does not change. 


\section{Difference Engine Simulation: Age Groups and Domains}

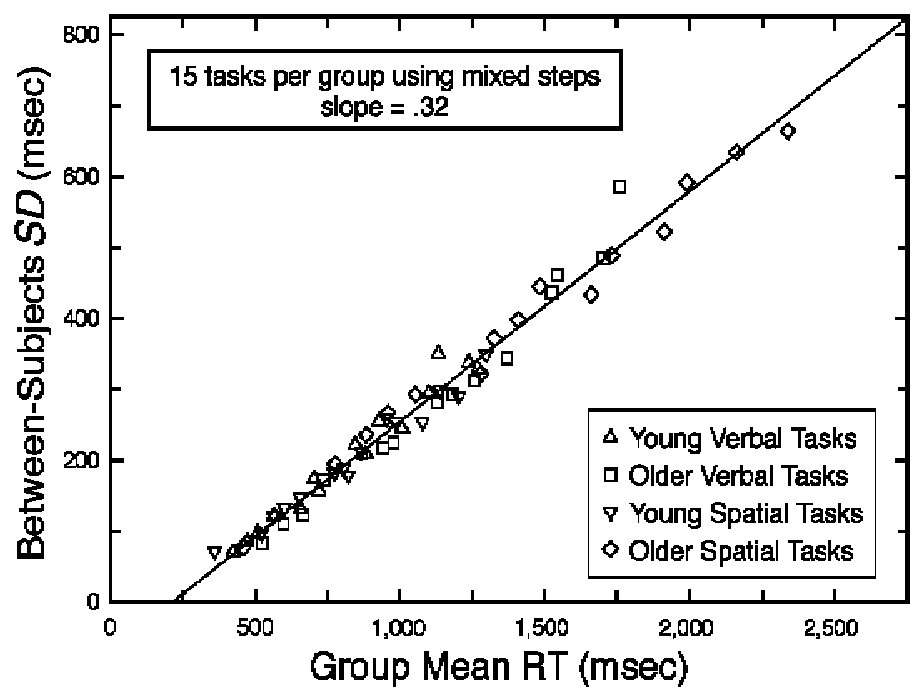

Figure 12. Between-subjects standard deviation $(S D)$ plotted as a function of group mean response time (RT). Data were generated by computer simulation (see text). The graph presents data from simulated verbal and visuospatial tasks consisting of a mixture of step durations. The older adult simulations assumed that older verbal processing steps were $50 \%$ longer than young verbal processing steps, whereas older visuospatial processing steps were twice as long as young visuospatial processing steps. Note that to facilitate comparisons, we made the ratio of the scale of the $x$-axis to the scale of the $y$-axis the same as in Figure 5.

Furthermore, because the equation for the regression of individual and subgroup RTs on group mean RTs follows directly from the regression of $S D$ on mean RT, the cognitive slowing coefficient does not enter into the relationship between the RTs of an individual or subgroup and the mean RTs for their age group. That is, it follows from Equation 12 that

$$
\mathrm{RT}_{i}=\left(1+z_{i} m\right) \mathrm{RT}_{\mathrm{avg}}-z_{i} m s_{r} t_{r} .
$$

As revealed by Equation 13, the difference engine makes the surprising prediction that the slope of the regression of the RTs of fast and slow individuals and subgroups on the RTs of their peers depends on their $z$ score relative to their age group but is otherwise independent of both age and domain. In fact, the only difference between Equation 13, the general equation for all adult age groups, and Equation 10, the corresponding equation for young adults, is in the intercept that in the general equation contains an age-specific sensorimotor slowing term $\left(s_{r}\right)$.

Computer simulations of the difference engine that relaxed some of the assumptions of the mathematical model were used to test the prediction (Equation 12) that the slope of the regression of $S D$ on mean RT is independent of both age and domain as well as the related prediction regarding the regression of fast and slow RTs on average RTs (Equation 13). To assess the robustness of the model, we simulated groups of 100 young and 100 older adults performing 30 verbal and 30 visuospatial tasks (one young and one older group for each domain). Within each domain, these tasks consisted of $3,4,5,6,7$, or 8 steps, with five tasks at each number of steps. For young adults, each task was made up of a randomly selected mixture of 20-, 50-, 100-, and 200-msec step durations. The results for the visuospatial simulations were presented previously in Figure 9 (see lower left panel), and thus for present purposes, we simulated another 30 verbal tasks using the same target values as those in previous simulations.

Both young and older adults were simulated using tasks with the same composition, but the degree of slowing was different for the two domains. For example, if the easiest visuospatial task had three steps with mean durations of 20,20 , and $50 \mathrm{msec}$ for young adults, then the durations of the steps for that task were 40, 40, and 100 msec for older adults. Likewise, if the easiest verbal task had three steps of 20,50, and $200 \mathrm{msec}$ for young adults, then the durations of those steps were 30,75 , and 300 for older adults. Thus, older adults were simulated to be $50 \%$ slower on verbal tasks and $100 \%$ slower on visuospatial tasks, consistent with Hale and Myerson (1996) and Lima et al. (1991).

As may be seen in Figure 12, the simulations resulted in very similar relationships between $S D$ and mean RT regardless of the age group or cognitive domain being simulated. Each simulated age group was then divided into four quartiles on the basis of individual speed factors, and 


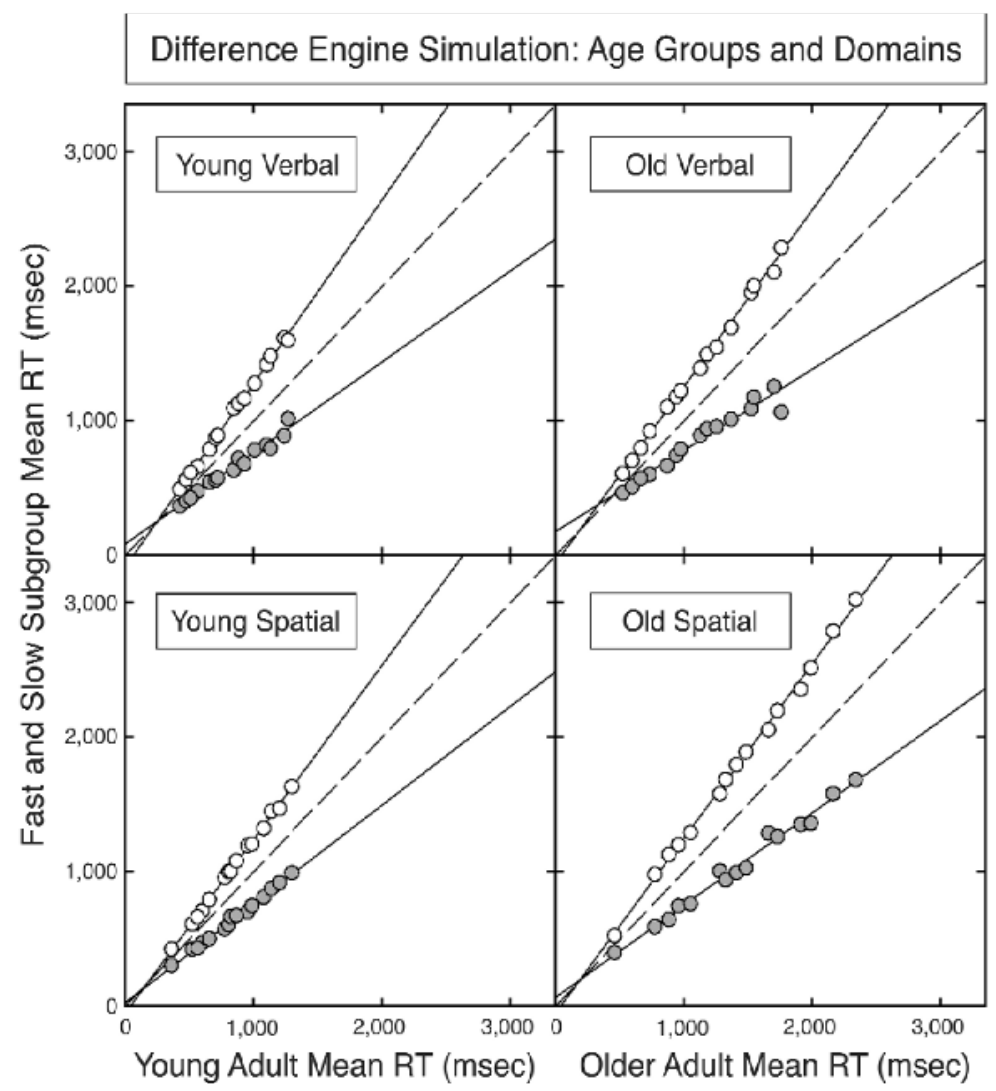

Figure 13. Response times (RTs) of fast and slow subgroups plotted as a function of the group mean RTs. Data were taken from the same simulation as in Figure 12. The left panels show simulated data from young adults, and the right panels show simulated data from older adults. Fast subgroups are represented by gray circles; slow subgroups are represented by white circles.

the top and bottom quartiles were taken as the slow and fast subgroups, respectively. Figure 13 shows the regressions of subgroup RTs on the corresponding mean RTs for each age group in each domain. In both figures, for the sake of clarity and also so as to depict the same number (i.e., 15) of task conditions as in comparable human data from Hale and Myerson (1996), only data from the oddnumbered tasks from each domain are shown.

As predicted by the mathematical model, the regression lines for the simulated slow subgroup RTs (fit to the white circles) were highly similar regardless of age or domain, and the same was true for simulated fast subgroup regression lines (fit to the gray circles). Notably, the performance of simulated individual subjects was fairly consistent across task conditions. On average, the simulated young subjects in the fast and slow subgroups were faster or slower than average in 13.6 out of 15 conditions, whereas the simulated fast and slow older subjects were faster or slower than average in 13.5 conditions.

The simulation results demonstrate that the difference engine's predictions regarding the effects (or the lack thereof) of age and domain do not depend on the assumption of equal mean step durations that expedited the math- ematical development, but appear to be more robust and hold for tasks that involve different mixtures of step durations as well. More specifically, regardless of variability in step durations, the model predicts that neither the relationship between $S D$ and group mean RT nor the relationship between subgroup RT and group mean RT will be substantially affected by the age group or by the domain involved, even if different domains involve different degrees of age-related slowing.

In order to test these predictions, we conducted a reanalysis of data from Hale and Myerson (1996). Figure 14 depicts the $S D$ s of both the 24 young and the 24 older adults on both the four verbal and the four visuospatial RT tasks (15 task conditions in each domain), and Figure 15 depicts the performance of fast and slow subgroups in both domains. The subgroups were selected to be the fastest and slowest 6 individuals in each age group (i.e., the first and fourth quartiles), determined using the same mean $z$-score procedure as Hale and Jansen (1994) and Hale and Myerson (1993).

As may be seen in Figure 14, there was an approximately linear relation between the $S D$ and group mean RT for both age groups in both the verbal and visuospatial domains. The 


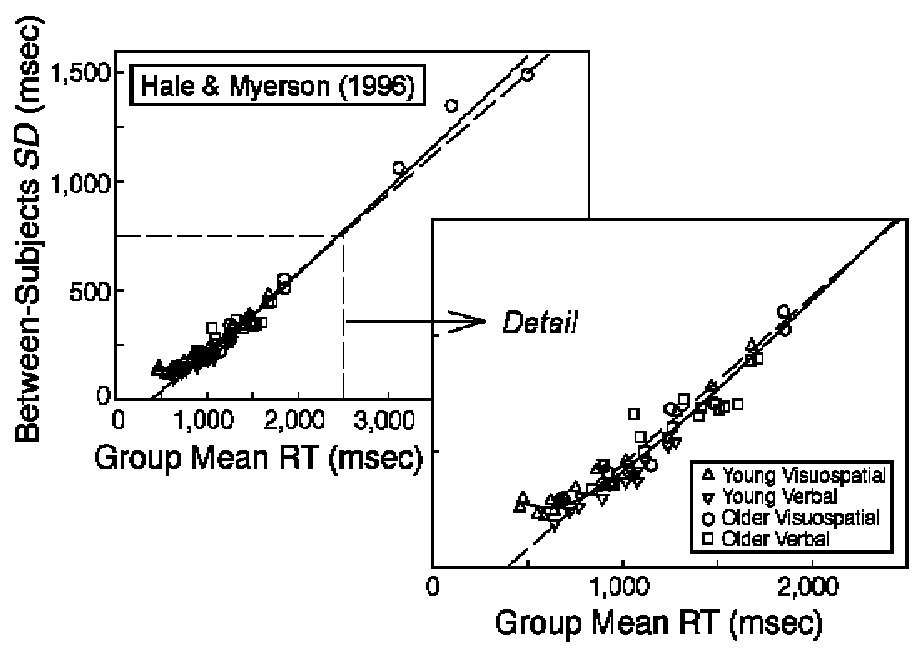

Figure 14. Between-subjects standard deviations ( $S D$ s) of young and older adult groups plotted as a function of their respective group mean response times (RTs). Data are taken from Hale and Myerson (1996). Each point represents performance by one age group in one experimental condition. In the panel labeled "Detail," the data are replotted over a truncated range in order to make it easier to resolve individual data points. Note that to facilitate comparisons with previous figures, we made the ratio of the scale of the $x$-axis to the scale of the $y$-axis the same as in Figure 5 .

solid curve is the theoretical function based on Equation 7, and the dashed line is the regression line. Tests over the same range of RTs revealed no significant differences either between the verbal and visuospatial regressions or between the regressions for the two age groups. These findings provide support for the present model because they are predicted directly by the fact that the cognitive slowing coefficient cancels out in the derivation of Equation 11 (which describes the relation between group $S D$ and mean RT).

As may be seen in Figure 15, the RTs of the fast and slow older adult subgroups were linear functions of average older adult RTs. Moreover, these relations were virtually collinear with those for the corresponding subgroups of young adults. That is, there were no significant age differences either between the regression parameters for the older and young fast subgroups or between the regression parameters for the older and young slow subgroups. Most importantly, equivalent results were observed in both the verbal and visuospatial domains despite the much greater degree of age-related slowing in the visuospatial domain. That is, there were no significant differences either between the regression parameters for the older fast verbal and fast spatial subgroups or between the regression parameters for older slow verbal and slow spatial subgroups. These findings provide further support for the present model because they are predicted directly by the fact that the cognitive slowing coefficient cancels out of the relation between older adult individual RTs and average performance for an older adult group (Equation 13). As a consequence, the relations between individual (or subgroup) and average RTs are not affected by differences in the degree of age-related slowing due to either age or domain.
Importantly, the subgroup data from Hale and Myerson (1996) presented in Figure 15 accurately reflect the patterns of fast and slow performances observed at the individual level. This may be seen by comparing Figure 15 with Figure 16, which depicts data from individual fast and slow older adults as well as fast and slow young adults in each domain. In each case, subjects were selected so as to be representative of their quartile in terms of the consistency of their performance and also in terms of how well their data were fit by a straight line $\left(r^{2}\right)$. Overall, fast and slow young adults were faster or slower than average for their age group in 14.3 out of 15 task conditions, and fast and slow older adults were faster or slower than average in 14.0 task conditions.

The results of the present analyses of the Hale and Myerson (1996) data replicate the findings of Hale and her colleagues (Hale \& Jansen, 1994; Hale \& Myerson, 1993) with respect to the performance of subgroups of young adults on visuospatial tasks (Figure 1) as well as Zheng et al.'s (2000) findings regarding subgroups of both young and older adults on such tasks. The present results also extend these previous findings to both young and older adults' performance on verbal tasks. Importantly, the present results confirm the difference engine's prediction that the parameters of certain key relationships between various aspects of speeded performance are independent of the interactive effects of age and processing domain.

\section{DISCUSSION}

We have presented a model of diversity in speeded information processing. This model, the difference engine, 


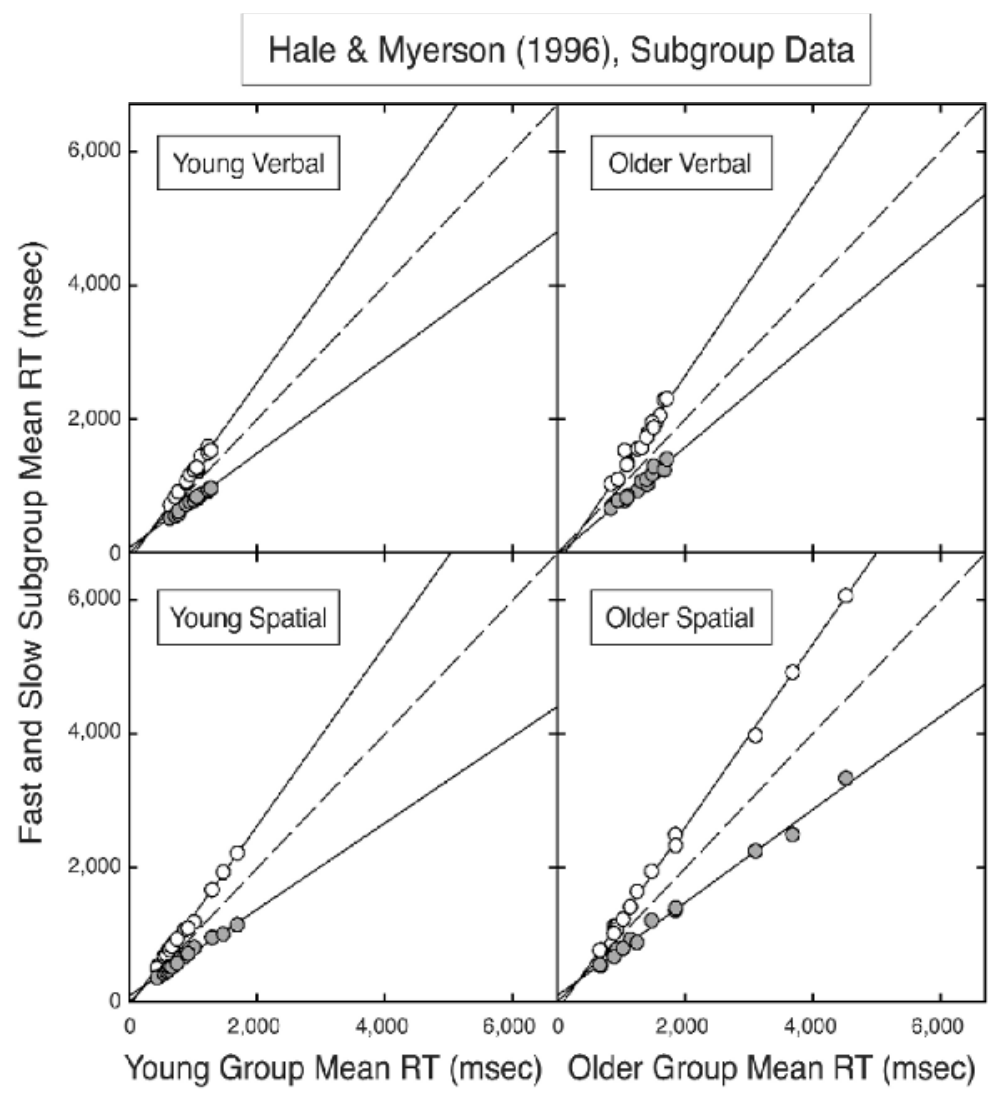

Figure 15. Response times (RTs) of fast and slow subgroups of young and older adults plotted as a function of their respective group mean RTs. Data are taken from Hale and Myerson (1996). Each point represents performance by a subgroup of one age group in one experimental condition.

assumes that for purposes of analyzing individual differences (including differences attributable to age and health status as well as differences in ability), information processing may be represented by a series of generic computational steps. Although an individual's processing steps may vary in duration, they are correlated in such a way that all of the processing steps of some individuals tend to be relatively brief whereas all of the processing steps of some other individuals tend to take longer than average. As a consequence of these intercorrelations, the diversity (as measured by the $S D$ for the group) of the cognitive component of RTs increases approximately linearly with task difficulty (as indexed by the average RT for the group). Because the duration of the cognitive component is uncorrelated with the response component, however, the relation between $S D$ and mean RT is actually positively accelerated.

The difference engine does more, however, than explain the form of the relation between $S D$ and mean RT. It also explains the approximately linear form of the regression of the RTs of an individual or subgroup on the RTs of their peers, and it provides specific interpretations of the parameters of such regressions. Finally, the model may be gen- eralized to differences in processing speed between groups that differ in age and health status, as well as to the interaction of such differences with individual differences in ability. This generalization follows directly from the assumption that although groups differ, on average, in processing speed, other relevant characteristics (i.e., the number of processing steps required to perform specific tasks as well as the intercorrelations between step durations) are unaffected by age and health status. Support for the difference engine appears to be quite broad in that, as predicted, the same pattern of results was observed in both young and older adults performing tasks from both the verbal and visuospatial domains, despite the fact these domains are differentially affected by age-related changes in processing speed.

\section{Comparative Human Cognition}

An important theme of the present effort, stripped of its empirical and theoretical specifics, is that there exist certain phenomena (all having to do with the magnitude of differences in speeded cognition) that share common properties even though they manifest themselves in traditionally distinct research areas (Hale, 1997). These phenomena sug- 


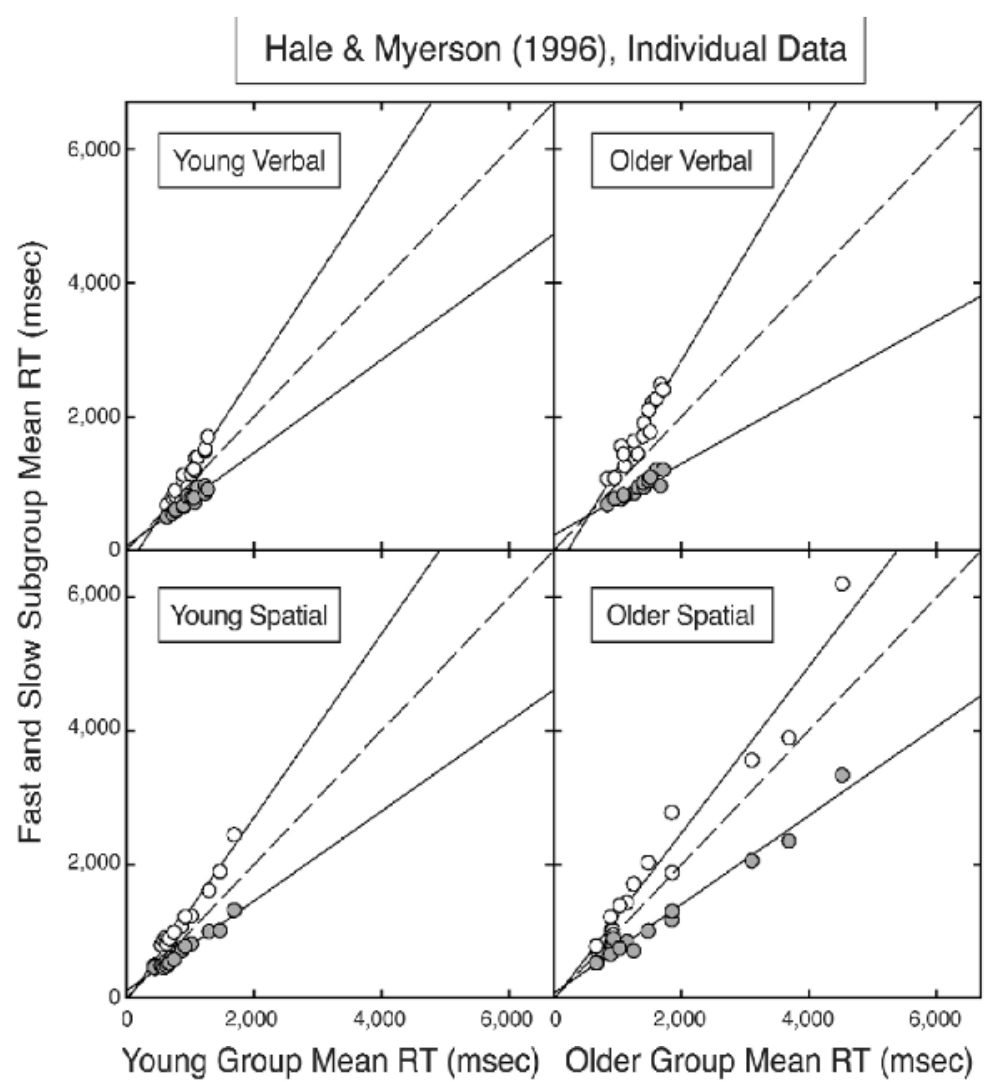

Figure 16. Response times (RTs) of representative fast and slow young and older individuals plotted as a function of their respective group mean RTs. Data are taken from Hale and Myerson (1996). Each point represents the performance of one individual in one experimental condition.

gest that there may be fundamental mechanisms underlying individual differences in information processing that operate in a similar fashion regardless of the cause of their operation (e.g., whether they are enabled by developmental or health-related events). The difference engine represents an attempt to model such fundamental mechanisms.

These mechanisms are hypothesized to underlie three sets of findings. First, when examined across a range of tasks that differ in difficulty, strikingly linear relations have been observed between the RTs of selected individuals (e.g., fast or slow subgroups) and the mean RTs for their peers (e.g., Hale \& Jansen, 1994; Zheng et al., 2000). Second, orderly linear relations have been observed between the RTs of different age groups (e.g., children and young adults or older and younger adults) tested on the same tasks (for a review, see Cerella \& Hale, 1994). Third, linear relations between group mean RTs have also been reported in experimental studies and meta-analyses that compared the RTs of various special populations with the RTs of control groups. Included among these studies are those that have examined individuals suffering from Alzheimer's disease (Myerson et al., 1998), brain injury (Ferraro, 1996), depression (White et al., 1997), and multiple sclerosis (Kail, 1997).
Similar findings in different populations suggest the possibility of similar explanations, and the difference engine represents one possible, quite general, explanation.In order to establish such generality, however, detailed analyses of individual performance will be needed in each case because, as is well known, relations between group averages do not necessarily reflect what is going on at the individual level. As a first step, examination of the relation between group $S D$, reflecting the diversity of individual performance, and mean RT, reflecting task difficulty, may prove quite useful.

For example, consider the data from a recent metaanalysis of the effects of depression on speeded cognitive performance (White et al., 1997). As may be seen in the upper panel of Figure 17, there is a linear relation between the RTs of depressed and control groups, which suggests that the depressed were approximately $30 \%$ slower than controls across a variety of information-processing tasks (e.g., choice RT, Stroop color naming, and delayed matching to sample). As may be seen in the lower panel, the relation between $S D$ and group mean RT was approximately linear for both the depressed and control groups. Notably, there were no significant group differences in either the slope or intercept of this relation, and for both groups, the 


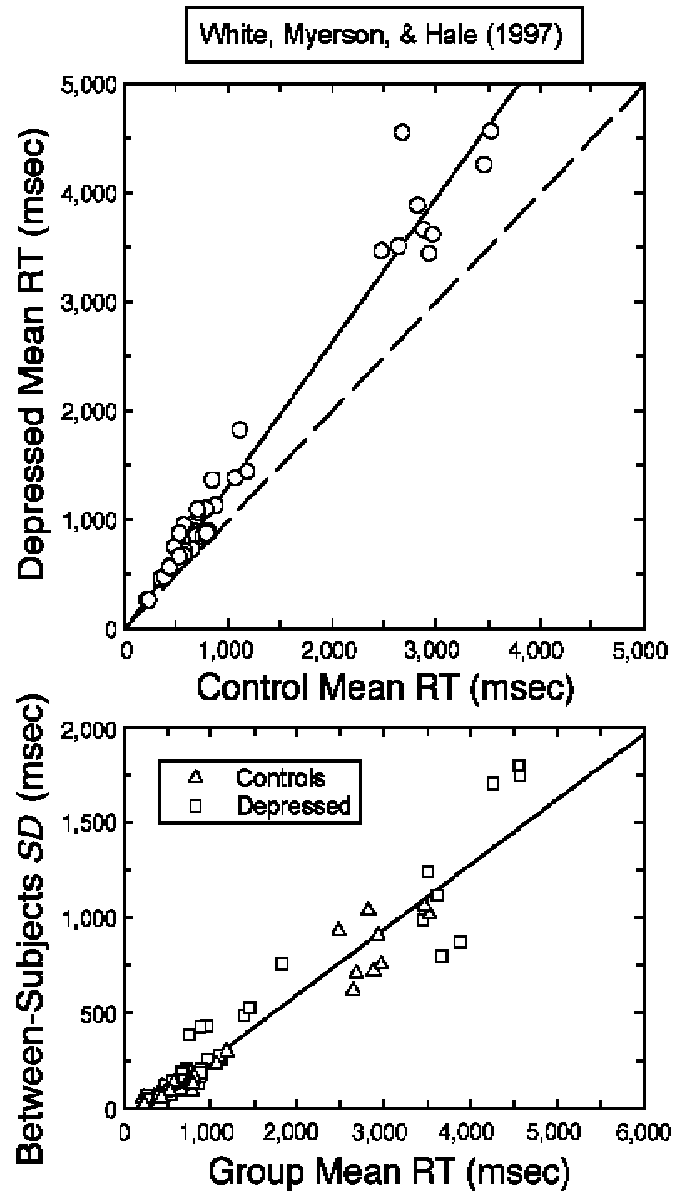

Figure 17. Mean response times (RTs) of depressed groups plotted as a function of the mean RT for healthy control groups (upper panel) and between-subjects standard deviation $(S D)$ plotted as a function of group mean RT for both depressed and control groups (lower panel). Data are taken from the metaanalyses reported by White, Myerson, and Hale (1997). In the upper panel, the solid line represents the regression of the RTs of depressed groups on the RTs of control groups. In the bottom panel, the solid line is the regression of $S D$ on mean RT based on the data from both depressed and control groups.

slopes and intercepts were similar to those for young and older adults (Figures 3 and 10). In all cases, the slope of the relation between $S D$ and mean was between .30 and .35 and the intercept was between -75 and $-100 \mathrm{msec}$. These results support the difference engine, of course, but more broadly, they are consistent with the idea that similar mechanisms may underlie the diversity of speeded performance in groups of depressed individuals and in groups of both young and older healthy individuals, and the same may be true for other populations as well.

There is a growing awareness among researchers that there may be common phenomena, research questions, and methodologicaland theoretical issues involved in what have been traditionally distinct research areas, each focused on the cognitive abilities of a distinct population (e.g., Cerella
\& Hale, 1994; Faust et al., 1999; Ferraro, 1996; Fisher \& Glaser, 1996; Kail, 1997; Nebes \& Brady, 1992; Schatz, 1998; White et al., 1997). This growing awareness suggests that a new research area may be emerging, one that focuses on what may be termed the study of comparative human cognition (Hale, 1997). The present theoretical effort exemplifies this trend toward the integration of findings regarding different aspects of individual and group differences.

\section{Comparisons With Other Approaches}

The goals of the present effort are both broad and unique. As a consequence, the opportunities to compare the present approach with previous theoretical models having similar aims are somewhat limited. Recently, however, three articles (Faust et al., 1999; Ratcliff, Spieler, \& McKoon, 2000; Zheng et al., 2000) have touched on some of the same basic theoretical issues considered here. We will consider these recent efforts following a brief discussion of the relevant older literature.

The basic approach taken here to the variability of speeded performance (i.e., that of decomposing task performance into a series of generic processing steps) is common in mathematical models (Luce, 1986; Townsend \& Ashby, 1983) and may be traced back to pioneering efforts by McGill (1963). However, the variability of interest in such models is typically the trial-to-trial variability of individual subjects (as reflected in RT distributions) rather than the diversity of performance across individuals and tasks. At least under certain circumstances, the $S D$ s of such distributions are linear functions of the mean RT (e.g., Hale, Fry, \& Jessie, 1993; Myerson \& Hale, 1993), raising the possibility that the two forms of variability in RTs may be more than superficially similar.

In fact, the relation of within-subjects $S D$ (or semiinterquartile range) to individual mean (or median) RT is surprisingly similar to the relation of between-subjects $S D$ to group mean RT in two respects. First, the relation tends to hold across tasks; second, it tends to hold across age groups (i.e., children, young adults, and older adults) with little or no change in the value of either slope or intercept (Hale et al., 1993; Myerson \& Hale, 1993). It is possible that trial-to-trial fluctuations in processing efficiency are analogous to variations in efficiency from individual to individual or from group to group (Smith, Poon, Hale, \& Myerson, 1988) in that such trial-to-trial fluctuations may also be based on correlated changes in the speed with which processing steps are executed. If so, then this analogy leads directly to application of the present models.

However, analysis of the trial-to-trial variability of RTs, unlike analysis of between-subjects variability, must consider the problem of sequential dependencies. Much of the variation in RTs across trials is due to adjustments in decision criteria following errors and to the effects of stimulus and/or response repetition (e.g., Smith \& Brewer, 1995; for a review, see Luce, 1986), whereas sequential dependencies are obviously not an issue with respect to variation across individuals. Because of this limitation on the analogy between individual differences and trial-to- 
trial fluctuations, we will postpone further consideration of the parallels between them to another occasion.

Psychometric approaches. The decomposition of RTs into processing steps has a direct psychometric analogue in the decomposition of test scores into performance on individual items or subtests. Despite the analogy between tests and tasks, the phenomena of interest from the psychometric perspective are usually quite different from those that are the focus here. In particular, the psychometric approach tends to focus on the relative performance of individuals (i.e., their positions in the overall distribution), and psychometric research on speed has been especially concerned with its relation to intelligence. In contrast, the difference engine is concerned with predicting the absolute size of differences between individual RTs, and we remain agnostic in regard to the relation between speed and intelligence.

Although the goals of psychometric research have been quite different from those of the present effort, the difference engine incorporates basic psychometric findings. The present assumption of a general cognitive speed factor and a separate sensorimotor factor is consistent with the results of psychometric studies (Buckhalt, Whang, \& Fischman, 1998; Carroll, 1991; Kranzler \& Jensen, 1991). Moreover, the assumption that age does not change the level of individuals' performance relative to their peers is consistent with the results of longitudinal psychometric research (Hertzog \& Schaie, 1986).

Information-processing approaches. The goals of the present model are obviously quite different from those of a chronometric approach (e.g., Posner, 1978; Sanders, 1990) that seeks to decompose processing into qualitatively different cognitive operations. In contrast, the primary goal of the difference engine is to predict regularities in performance across tasks that differ substantially with respect to the cognitive operations involved. For example, what is critically important about visual search and mental rotation from the chronometric perspective is that they obviously involve different cognitive processes, yet the present analyses suggest that individual differences in one process are predictable from individual differences in the other process. Moreover, aging appears to affect performance on both types of tasks (and other visuospatial tasks as well) to approximately the same degree. Equivalent age-related slowing of visual search and mental rotation was first shown in a multitask experiment in which the same older and young adults performed both types of tasks (Hale, Myerson, Faust, \& Fristoe, 1995). This finding has since been validated in a meta-analysis using standard regression techniques (Myerson, Adams, Hale, \& Jenkins, 2003) as well as with more refined meta-analytic methods based on hierarchical linear models (Sliwinski \& Hall, 1998).

This surprisingly robust finding suggests that regardless of whether individuals are slow because of age, ability, or both, being slow at one process means that one will tend to be slow at the other process. Thus, making a distinction between visual search and mental rotation, although essential for some purposes, would not contribute to the present effort. Indeed, the strength of the present approach is that it can reveal similarities precisely under conditions where differences in the underlying cognitive processes are known to exist. We raise these issues here in order to reemphasize the fact that our simplifying assumptions, which may conflict with some researchers' strongly held beliefs regarding the complicated nature of even simple information processing, need to be seen as the means to an end. That end is to reveal and understand large-scale, quantitative regularities in the patterns of individual and group differences. To achieve that end, the present model ignores processing distinctions that most cognitive psychologists accept as valid, thereby potentially sacrificing some degree of detail. Yet at the same time, the present model strives to provide detail that is missing from other accounts in that the difference engine is intended to predict both the magnitude and the distribution of individual performances.

Other general slowing models. In the areas of cognitive development and cognitive aging, the present theoretical effort is not alone in having sacrificed the usual task and process distinctions of cognitive psychology (e.g., Cerella, 1985; Kail, 1991). Of particular relevance are the models proposed by Cerella (1985) and Cerella and Hale (1994), who divided processing into two components, one cognitive and one sensorimotor, for purposes of analyzing age-related changes in RTs across the life span. The difference engine may be viewed as an extension of that approach to individual differences and to group differences attributable to factors other than age. This extension is important because such ideas are not a necessary part of a general slowing hypothesis, since general slowing could be peculiar to the effects of age or it could describe changes at the group level but not those at the individual level. To the best of our knowledge, Zheng et al. (2000) were the first to take an explicit position on this issue and to consider its theoretical implications. Their magnification hypothesis, like the difference engine, assumes that age-related slowing operates at the individual level, magnifying everyone's processing times and increasing the size of individual differences by the same task-independent factor. Their mathematical account was highly simplified, however, and they called for further theoretical work along the lines of the present effort.

The diffusion model. Ratcliff et al. (2000) have recently suggested applying the diffusion model (Ratcliff, 1978) to the effects of aging on RT. The diffusion model describes within-subjects RT distributions and speed/accuracy tradeoff functions, but it has not previously been used to study group differences. With respect to age-related differences, Ratcliff et al. suggested that what appear to be differences in processing speed may be due to differences in decision criteria (i.e., older adults may be more cautious rather than slower). This hypothesis, however, has not generally been supported. As Cerella (1990) showed, in fact, older adults tend to make more rather than fewer errors than young adults. Moreover, as Myerson et al. (2003) have pointed out, a number of recent studies have shown that older adults continue to process information more 
slowly than young adults even when stimulus-duration threshold procedures are used to eliminate accuracy differences (e.g., Mayr, Kliegl, \& Krampe, 1994, 1996; Zacks \& Zacks, 1993).

In contrast to the difference engine, the diffusion model makes no assumptions or predictions regarding the covariation between individual RTs on different tasks, nor does it predict or explain the form of the relation between $S D$ and mean RT. As Ratcliff et al. (2000) have specifically pointed out, the diffusion model can accommodate increases in between-subjects $S D$ as a function of the mean, but it does not predict them. Moreover, the diffusion model does not predict the approximately linear form of the regression of the RTs of an individual or subgroup on the RTs of their peers (although again, the diffusion model is not inconsistent with such results), and it provides no specific interpretations of the parameters of such relations. Thus, although the diffusion model is not inconsistent with the results of the analyses reported here, its treatment of the present findings would be necessarily post hoc. For example, the simulation of subgroup differences in Ratcliff et al. merely shows that there exist some values for the diffusion model's parameters that emulate actual data. However, Ratcliff et al. presented no principled treatment of the way in which parameters do (or do not) change across tasks, and thus they failed to come to grips with the core of the theoretical question posed by the data.

The rate-amount model. Faust et al. (1999) have proposed what they term a rate-amount model that predicts linear relationships between the RTs of individuals and groups and a linear relation between $S D$ and mean RT. The difference engine, too, may be thought of as a rate-amount model, in which the amount of processing required to reach a decision is represented by the number of processing steps and individuals and groups differ in the rate at which these steps are completed. Despite many similarities, the two models differ in a number of respects, including some of the fundamental assumptions and the mathematical approach taken. These differences, in part, reflect the different goals of the two efforts. The difference engine is an explicitly theoretical model of the large-scale structure of individual and group differences in informationprocessing speed, whereas the rate-amount model is intended to provide a basis for statistical testing and isolation of small-scale group differences, assuming that such a large-scale structure exists.

In terms of generating predictions, it is important to note that the model proposed by Faust et al. (1999) is explicitly a one-compartment model, although they assume two separate individual difference variables, one governing speed and the other governing amount. In contrast, the difference engine is a two-compartment model that distinguishes response selection and execution from cognitive processing. The difference engine then further subdivides the cognitive compartment into generic processing steps with equivalent correlations between step durations. Although the two models make a number of predictions in common, as a consequence of these differences, only the difference engine correctly predicts the initial positive acceleration of the approximately linear relation between $S D$ and mean RT. The difference engine also provides an interpretation of the $x$-intercept of the linear approximation of this relation in terms of the time required for response selection and execution. Additionally, this interpretation-which the rate-amount model specifically eschews (Faust et al., 1999) - is consistent with observed estimates and correctly predicts the approximate point at which the regression of individualor subgroup RTs on group mean RT will intersect the equality diagonal.

Faust et al. (1999) have suggested that their rate-amount model provides a statistical basis for asking important questions such as whether all younger adults become slowed to the same degree as they grow older. Their model, however, does not predict what the answer to this question will be. In contrast, the difference engine does propose an answer to this question as well as other related questions. Our model specifically proposes that at least within broad cognitive domains (e.g., verbal and visuospatial tasks), age-related changes effectively multiply the speed coefficients of all individuals, fast or slow, by an equivalent factor, without affecting other aspects of processing. On the basis of this theoretical assumption, the difference engine makes the unique prediction that differences in slowing factors between domains or age groups will not affect other key relationships. That is, such slowing factors will affect neither the slope of the relation of the group's $S D$ to mean RT nor the relationships of individual and subgroup RTs to the mean RT for their age group. The results of the present analyses of the Zheng et al. (2000) and Hale and Myerson (1996) data strongly support these unique predictions.

It may be noted that the precise details of the theoretical machinery that are the focus of the difference engine may be largely irrelevant for the statistical purposes that Faust et al. have in mind. The major goal of their effort was to justify transforms that could facilitate the isolation of small-scale group differences. The justification of these transforms depends on linear or approximately linear relations between group $S D$ s and individual RTs, and linear relations between group mean RTs. The two models are in agreement as to the approximate form of these relations, and thus we see the efforts as complementary, one more directed to solving theoretical problems in large-scale structure and the other more directed to solving the statistical problems raised by the existence of such a structure. Whereas the transformations justified by the rate-amount model may facilitate the identification of small-scale group differences, the parameters of the equations for individual performance (Equations 10 and 13), easily interpretable in terms of individual $z$ scores, may facilitate the identification of clinically significant individual differences (Schatz, Hale, \& Myerson, 1998).

\section{Conclusion}

The most obvious potential limitation of the difference engine model is that it relies on strong, simplifying assumptions, although computer simulations suggest that the model's predictions are quite robust when some, but not all, of these assumptions are relaxed. The important 
question with regard to the difference engine, of course, is not whether the model's assumptions are precisely true. They are not. They are merely simplifying assumptions intended to facilitate the mathematical development so that we can examine the consequences of certain theoretical ideas. Rather, the important question with respect to the difference engine is whether it leads to an increase in the ability to predict performance and to new insights into why performance conforms to these predictions.

With respect to the accuracy of the difference engine's predictions, the results of a number of new analyses of previously published data provide considerable support for the present model. These analyses examined the intercorrelations and factor structure of RTs as well as their means and $S D$ s, the relationship between these variables, and the relations between the RTs of individuals and groups that differ in age or ability. With respect to the insight gained from the model, the difference engine's most important contribution may be to provide theoretical linkage between these seemingly disparate phenomena. Taken together, the findings to date suggest that the theoretical model presented here provides a uniquely integrated account of individual and group differences on speeded cognitive tasks. Future research will be needed to establish the generality of the difference engine's predictions and their applicability to additional populations, including children and individuals with various health conditions.

\section{REFERENCES}

Buckhalt, J. A., Whang, P. S., \& Fischman, M. G. (1998). Reaction time and movement time relationships with intelligence in three different simple tasks. Personality \& Individual Differences, 24, 493-497.

CARroll, J. B. (1991). No demonstration that $g$ is not unitary, but there's more to the story. Intelligence, 15, 423-436.

Cerella, J. (1985). Information processing rates in the elderly. Psychological Bulletin, 98, 67-83.

Cerella, J. (1990). Aging and information-processing rate. In J. E. Birren \& K. W. Schaie (Eds.), Handbook of the psychology of aging (3rd ed., pp. 201-221). San Diego: Academic Press.

Cerella, J. (1994). Generalized slowing in Brinley plots. Journals of Gerontology, 49, P65-P71.

Cerella, J., \& Hale, S. (1994). The rise and fall in informationprocessing rates over the life span. Acta Psychologica, 86, 109-197.

Dunn, J. C., \& Kirsner, K. (1988). Discovering functionally independent mental processes: The principle of reversed association. Psychological Review, 95, 91-101.

Faust, M. E., Balota, D. A., Sieleler, D. H., \& Ferraro,F. R. (1999). Individual differences in information processing rate and amount: Implications for group differences in response latency. Psychological Bulletin, 125, 777-799.

Ferraro, F. R. (1996). Cognitive slowing in closed-head injury. Brain \& Cognition, 32, 429-440.

Fisher, D. L., \& GLASER, R. A. (1996). Molar and latent models of cognitive slowing: Implications for aging, dementia, depression, development, and intelligence. Psychonomic Bulletin \& Review, 3, 458-480.

Gorsuch, R. L. (1983). Factor analysis (2nd ed.). Hillsdale, NJ: Erlbaum.

Hale, S. (1997). Introduction. [Special issue: Comparative adult cognition]. Aging, Neuropsychology, \& Cognition, 4, 155-156.

Hale, S., Fry, A. F., \& JeSsie, K. A. (1993). Effects of practice on speed of information processing in children and adults: Age-sensitivity and age-invariance. Developmental Psychology, 29, 880-892.

HALE, S., \& JANSEN, J. (1994). Global processing-time coefficients characterize individual and group differences in cognitive speed. Psychological Science, 5, 384-389.

HALe, S., \& Myerson, J. (1993, November). Ability-related differences in cognitive speed: Evidence for global processing-time coefficients. Poster presented at the annual meeting of the Psychonomic Society, Washington, DC.

Hale, S., \& Myerson, J. (1996). Experimental evidence for differential slowing in the lexical and nonlexical domains. Aging, Neuropsychology, \& Cognition, 3, 154-165.

Hale, S., Myerson, J., Faust, M., \& Fristoe, N. (1995). Converging evidence for domain-specific slowing from multiple nonlexical tasks and multiple analytic methods. Journals of Gerontology, 50B, P202P211.

Hale, S., Myerson, J., Smith, G. A., \& Poon, L. W. (1988). Age, variability, and speed: Between-subjects diversity. Psychology \& Aging, 3, 407-410.

Hale, S., Myerson, J., \& Wagstaff, D. (1987). General slowing of nonverbal information processing: Evidence for a power law. Journal of Gerontology, 42, 131-136.

Hertzog, C., \& Schaie, K. W. (1986). Stability and change in adult intelligence: 1. Analysis of longitudinal covariance structures. Psychology \& Aging, 1, 159-171.

KAIL, R. (1991). Developmental change in speed of processing during childhood and adolescence. Psychological Bulletin, 109, 490-501.

KAIL, R. (1992). General slowing of information-processing by persons with mental retardation. American Journal on Mental Retardation, $\mathbf{9 7}$ 333-341.

KaIL, R. (1997). The neural noise hypothesis: Evidence from processing speed in adults with multiple sclerosis. Aging, Neuropsychology, \& Cognition, 4, 157-165.

Kranzler, J. H., \& Jensen, A. R. (1991). The nature of psychometric $g$ : Unitary process or a number of independent processes? Intelligence, 15, 397-422.

Lawrence, B., Myerson, J., \& Hale, S. (1998). Differential decline of verbal and visuospatial processing speed across the adult life span. Aging, Neuropsychology, \& Cognition, 5, 129-146.

Lima, S. D., Hale, S., \& Myerson, J. (1991). How general is general slowing? Evidence from the lexical domain. Psychology \& Aging, 6, 416-425.

LuCE, R. D. (1986). Response latencies: Their role in inferring elementary mental organization. New York: Oxford University Press.

Mayr, U., Kliegl, R., \& Krampe, R. (1994). Time-accuracy functions for determining process and person differences: An application to cognitive aging. Cognitive Psychology, 26, 134-164.

Mayr, U., Kliegl, R, \& Krampe, R. (1996). Sequential and coordinative processing dynamics in figural transformations across the life span. Cognition, 59, 61-90.

McClelland, J. L. (1979). On the time relations of mental processes: An examination of systems of processes in cascade. Psychological Review, 86, 287-330.

McGill, W. J. (1963). Stochastic latency mechanisms. In R. D. Luce, R. R. Bush, \& E. Galanter (Eds.), Handbook of mathematical psychology (Vol. 1, pp. 309-360). New York: Wiley.

Myerson, J., Adams, D. R., Hale, S., \& Jenkins, L. (2003). Analysis of group differences in processing speed: Brinley plots, Q-Q plots, and other conspiracies. Psychonomic Bulletin \& Review, 10, 224-237.

Myerson, J., \& Hale, S. (1993). General slowing and age-invariance in cognitive processing: The other side of coin. In. J. Cerella, W. Hoyer, J. Rybash, \& M. Commons (Eds.), Adult information processing: Limits on loss (pp. 115-141). San Diego: Academic Press.

Myerson, J., Lawrence, B., Hale, S., Jenkins, L., \& Chen, J. (1998). General slowing of lexical and nonlexical information processing in dementia of the Alzheimer type. Aging, Neuropsychology, \& Cognition, 5, 129-146.

Nebes, R. D., \& Brady, C. B. (1992). Generalized cognitive slowing and severity of dementia in Alzheimer's disease: Implications for the interpretation of response-time data. Journal of Clinical \& Experimental Neuropsychology, 14, 317-326.

Posner, M. I. (1978). Chronometric explorations of mind: The third Paul M. Fitts lectures. Hillsdale, NJ: Erlbaum.

Ratcliff, R. (1978). A theory of memory retrieval. Psychological Review, 85, 59-108.

Ratcliff, R., Spieler, D., \& McKoon, G. (2000). Explicitly modeling the effects of aging on response time. Psychonomic Bulletin \& Review, 7, 1-25. 
SANDERS, A. F. (1990). Issues and trends in the debate on discrete vs. continuous processing of information. Acta Psychologica, 74, 123-167.

Schatz, J. (1998). Cognitive processing eff iciency in schizophrenia: Generalized versus domain-specific deficits. Schizophrenia Research, 30, 41-49.

Schatz, J., Hale, S., \& Myerson, J. (1998). Cerebellar contributions to linguistic processing efficiency revealed by focal damage. Journal of the International Neuropsychological Society, 4, 491-501.

SLIWINSKI, M. J., \& HALl, C. B. (1998). Task-dependent cognitive slowing in the elderly: A meta-analysis using hierarchical linear models with random coefficients. Psychology \& Aging, 13, 164-175.

Smith, G. A., \& Brewer, N. (1995). Slowness and age: Speed-accuracy mechanisms. Psychology \& Aging, 10, 238-247.

Smith, G. A., Poon, L. W., Hale, S., \& Myerson, J. (1988). A regular relationship between old and young adults' latencies on their best, average and worst trials. Australian Journal of Psychology, 40, 195-210.

Teichner, W. H., \& Krebs, M. J. (1974). Laws of visual choice reaction time. Psychological Review, 81, 75-98.

Townsend, J. T., \& AshBy, F. G. (1983). The stochastic modeling of elementary psychological processes. New York: Cambridge University Press.
Vernon, P. A. (1983). Speed of information processing and general intelligence. Intelligence, 7, 53-70.

Vernon, P. A., \& Jensen, A. R. (1984). Individual and group differences in intelligence and speed of information processing. Personality \& Individual Differences, 5, 411-423.

Vernon, P. A., Nador, S., \& Kantor, L. (1985). Reaction times and speed of information-processing: Their relationship to timed and untimed measures of intelligence. Intelligence, 9, 357-374.

White, D. A., Myerson, J., \& Hale, S. (1997). How cognitive is psychomotor slowing in depression? Aging, Neuropsychology, \& Cognition, 4, 166-174.

ZACKS, J. L., \& ZACKS, R. T. (1993). Visual search times assessed without reaction times: A new method and an application to aging. Journal of Experimental Psychology: Human Perception \& Performance, 19, 798-813.

Zheng, Y., Myerson, J., \& Hale, S. (2000). Age and individual differences in visuospatial processing speed: Testing the magnification hypothesis. Psychonomic Bulletin \& Review, 7, 113-120.

(Manuscript received June 1, 1999;

revision accepted for publication April 30, 2002.) 